\title{
LOCAL POINTWISE A POSTERIORI GRADIENT ERROR BOUNDS FOR THE STOKES EQUATIONS
}

\author{
ALAN DEMLOW AND STIG LARSSON
}

Abstract. We consider the standard Taylor-Hood finite element method for the stationary Stokes system on polyhedral domains. We prove local a posteriori error estimates for the maximum error in the gradient of the velocity field. Because the gradient of the velocity field blows up near reentrant corners and edges, such local error control is necessary when pointwise control of the gradient error is desirable. Computational examples confirm the utility of our estimates in adaptive codes.

\section{INTRODUCTION}

We consider finite element methods for the stationary Stokes equations

$$
\begin{aligned}
-\Delta \mathbf{u}+\nabla p & =\mathbf{f}, & & \text { in } \Omega, \\
\nabla \cdot \mathbf{u} & =g, & & \text { in } \Omega, \\
u & =0, & & \text { on } \partial \Omega .
\end{aligned}
$$

Here we assume that $\Omega \subset \mathbb{R}^{n}, n=2,3$, is a polygonal $(n=2)$ or polyhedral $(n=3)$ domain with Lipschitz boundary. Many of our main arguments also extend to polyhedral domains with non-Lipschitz boundaries such as cracks, but we assume Lipschitz domains for simplicity; see Remark 4 below. We assume that $f \in\left(L_{\infty}(\Omega)\right)^{n}$ and $g \in W_{q}^{1}(\Omega)$ for some $q>n$; in some of our results we assume further restrictions on $g$ when $n=3$. Finally, we also require that $\int_{\Omega} g \mathrm{~d} x=0$ in order to ensure existence and $\int_{\Omega} p \mathrm{~d} x=0$ in order to ensure uniqueness of solutions. With

$$
V=\left(H_{0}^{1}(\Omega)\right)^{n}, \quad X=L_{2}(\Omega),
$$

we introduce the bilinear form

$$
\mathcal{L}((\mathbf{u}, p),(\mathbf{v}, \lambda))=a(\mathbf{u}, \mathbf{v})+b(\mathbf{v}, p)-b(\mathbf{u}, \lambda),
$$

where

$$
a(\mathbf{u}, \mathbf{v})=\int_{\Omega} \sum_{i, j=1}^{n} \frac{\partial \mathbf{u}_{i}}{\partial x_{j}} \frac{\partial \mathbf{v}_{i}}{\partial x_{j}} \mathrm{~d} x, \quad b(\mathbf{v}, p)=-\int_{\Omega}(\nabla \cdot \mathbf{v}) p \mathrm{~d} x
$$

Received by the editor May 10, 2010 and, in revised form, August 12, 2011.

2010 Mathematics Subject Classification. Primary 65N30.

Key words and phrases. Stokes system, Taylor-Hood, finite element, a posteriori, gradient, local.

The first author was partially supported by NSF grant DMS-0713770.

The second author was partially supported by the Swedish Research Council (VR) and by the Swedish Foundation for Strategic Research (SSF) through GMMC, the Gothenburg Mathematical Modelling Centre. 
Writing also

$$
(\mathbf{f}, \mathbf{v})=\int_{\Omega} \sum_{i, j=1}^{n} \mathbf{f}_{i} \mathbf{v}_{i} \mathrm{~d} x, \quad(g, \lambda)=\int_{\Omega} g \lambda \mathrm{d} x,
$$

we obtain the weak formulation of (1.1): find $(\mathbf{u}, p) \in V \times X$ such that

$$
\mathcal{L}((\mathbf{u}, p),(\mathbf{v}, \lambda))=(\mathbf{f}, \mathbf{v})+(g, \lambda) \quad \forall(\mathbf{v}, \lambda) \in V \times X .
$$

Let $\left\{\mathcal{T}_{h}\right\}$ be a regular family of triangulations of $\Omega$, generated for example by an adaptive bisection algorithm. We assume that $V_{h} \subset V$ and $X_{h} \subset X$ are standard Taylor-Hood finite element spaces corresponding to a mesh $\mathcal{T}_{h}$; properties are given below. The finite element method for (1.1) is: find $\left(\mathbf{u}_{h}, p_{h}\right) \in V_{h} \times X_{h}$ such that

$$
\mathcal{L}\left(\left(\mathbf{u}_{h}, p_{h}\right),\left(\mathbf{v}_{h}, \lambda_{h}\right)\right)=\left(\mathbf{f}, \mathbf{v}_{h}\right)+\left(g, \lambda_{h}\right) \quad \forall\left(\mathbf{v}_{h}, \lambda_{h}\right) \in V_{h} \times X_{h} .
$$

We enforce $\int_{\Omega} p_{h} \mathrm{~d} x=0$ in order to ensure uniqueness.

Our goal in this paper is to prove local a posteriori error estimates for $\nabla\left(\mathbf{u}-\mathbf{u}_{h}\right)$ in the maximum norm. More precisely, let $D \subset \Omega$ be a given target subdomain. We seek a posteriori control of

$$
\left\|\nabla\left(\mathbf{u}-\mathbf{u}_{h}\right)\right\|_{L_{\infty}(D)}=\sup _{x \in D} \max _{i, j}\left|D_{i}\left(\mathbf{u}_{j}-\mathbf{u}_{h, j}\right)(x)\right|
$$

along with the related quantity $\left\|p-p_{h}\right\|_{L_{\infty}(D)}$.

In order to motivate our results, we briefly describe the Ph.D. thesis [Sve06] by E. D. Svensson. It considers the problem of computationally characterizing mixing in incompressible flows. Let $\Omega \subset \mathbb{R}^{n}, n=2,3$, be an open set containing a fluid. The motion $\mathbf{v}:[0, \infty) \times \Omega \rightarrow \Omega$ of the fluid is described by the system

$$
\frac{\partial \mathbf{v}(t, x)}{\partial t}=\mathbf{u}(\mathbf{v}(t, x)), t>0 ; \quad \mathbf{v}(0, x)=x,
$$

of ordinary differential equations. Here $x \in \Omega$ is the starting point of the particle path and $\mathbf{u}$ is the velocity field obtained by solving (1.1). In order to solve (1.5) computationally, Svensson discretized both (1.5) and (1.1) by the finite element method. In order to control the error in approximating (1.5), it is necessary to control $\left\|\mathbf{u}-\mathbf{u}_{h}\right\|_{L_{\infty}(\Omega)}$. Such bounds can be found for arbitrary polyhedral domains in $\mathbb{R}^{2}$ or $\mathbb{R}^{3}$ in SL06.

Svensson also developed a shadowing error estimate for (1.5), that is, an estimate for the distance between the computed path and a true path not necessarily having the same starting point. This estimate involves a linearization of (1.5), which in turn requires pointwise error bounds for $\nabla\left(\mathbf{u}-\mathbf{u}_{h}\right)$. Global pointwise gradient bounds for the case when $\Omega$ is convex can be found in SL06. However, (1.5) is often naturally formulated in nonconvex domains, and $\nabla \mathbf{u}$ is generally not bounded near reentrant corners and edges of $\partial \Omega$. For this application, it is sufficient to provide a posteriori control of $\left\|\nabla\left(\mathbf{u}-\mathbf{u}_{h}\right)\right\|_{L_{\infty}(D)}$ on regions $D \subset \Omega$ not abutting nonconvex parts of $\partial \Omega$. In this work we employ techniques developed in Dem07 in the context of Poisson's problem in order to provide local pointwise gradient error control for the Stokes system. We note that while Dem07] provides a roadmap for the current work, our proofs here also involve significant technical challenges not present in scalar elliptic problems.

More precisely, let $D \subset \Omega$ with $D$ lying a distance $d>0$ from any reentrant vertex (when $n=2,3$ ) or edge (when $n=3$ ) of $\partial \Omega$. We assume for simplicity that 
$d \leq 1$. Let $D_{d}=\{x \in \Omega: \operatorname{dist}(D, x)<d\}$. We define the $W_{\infty}^{1}$-type residual error indicator

$$
\begin{aligned}
\eta_{1, \infty}(T)= & h_{T}\left\|\mathbf{f}+\Delta \mathbf{u}_{h}-\nabla p_{h}\right\|_{L_{\infty}(T)} \\
& +\left\|\llbracket \nabla \mathbf{u}_{h} \rrbracket\right\|_{L_{\infty}(\partial T)}+\left\|g-\nabla \cdot \mathbf{u}_{h}\right\|_{L_{\infty}(T)}, \quad T \in \mathcal{T}_{h} .
\end{aligned}
$$

Here $\llbracket \nabla \mathbf{u}_{h} \rrbracket$ is the jump in the (componentwise) normal derivative of $\mathbf{u}_{h}$ across the element boundary $\partial T$ and $h_{T}=\operatorname{diam}(T)$. Also, let $\underline{h}=\min _{T \in \mathcal{T}_{h}} h_{T}$. Finally, let $\mathcal{T}_{D_{d}}=\left\{T \in \mathcal{T}_{h}: T \cap D_{d} \neq \emptyset\right\}$. We use the standard norms $\|\cdot\|_{L_{p}(D)},\|\cdot\|_{W_{p}^{m}(D)}$ and seminorms $|\cdot|_{W_{p}^{m}(D)},|\cdot|_{C^{1, \beta}(\bar{D})}$. Our first main result is then the following.

Theorem 1. Let $\rho \leq c_{0} \min \{d, \underline{h}\}$ for a sufficiently small constant $c_{0}$, and assume that $(\mathbf{u}, p) \in C^{1, \beta}\left(\overline{B_{\rho}}\right) \times C^{0, \beta}\left(\overline{B_{\rho}}\right)$ for some $\beta \in(0,1)$. Assume also that the Green's functions estimates of Lemma 10 below for convex polyhedral domains hold. Under the above assumptions, we then have

$$
\begin{aligned}
& \left\|\nabla\left(\mathbf{u}-\mathbf{u}_{h}\right)\right\|_{L_{\infty}(D)}+\left\|p-p_{h}\right\|_{L_{\infty}(D)} \\
& \leq C\left[\ln \frac{d}{\rho} \max _{T \in \mathcal{T}_{D_{d}}} \frac{h_{T}}{h_{T}+\operatorname{dist}(T, D)} \eta_{1, \infty}(T)\right. \\
& \left.+\frac{1}{d}\left(\left\|\mathbf{u}-\mathbf{u}_{h}\right\|_{L_{\infty}(\Omega)}+\max _{T \in \mathcal{T}_{h}} h_{T} \eta_{1, \infty}(T)\right)+\rho^{\beta}\left(|\mathbf{u}|_{C^{1, \beta}\left(\overline{B_{\rho}}\right)}+|p|_{C^{0, \beta}\left(\overline{B_{\rho}}\right)}\right)\right] .
\end{aligned}
$$

Here $B_{\rho}=B_{\rho}\left(x_{0}\right) \subset D_{d}$ is a ball of radius $\rho$ centered at a point where the maximum error is attained.

The above estimate consists of a local residual term, a regularization penalty, and a global pollution term. The local residual term $\max _{T \in \mathcal{T}_{D_{d}}} \frac{h_{T}}{h_{T}+\operatorname{dist}(T, D)} \eta_{1, \infty}(T)$ measures local error contributions from a neighborhood of the target region $D$. Since $\frac{h_{T}}{h_{T}+\operatorname{dist}(T, D)}=1$ when $T \cap D \neq \emptyset$, error contributions from $D$ are measured by the $W_{\infty}^{1}$-type residual indicator $\eta_{1, \infty}$. Error contributions from elements intersecting $D_{d}$ but not touching $D$ are measured by the term $\frac{h_{T}}{h_{T}+\operatorname{dist}(T, D)} \eta_{1, \infty}(T)$. The strength of this contribution decays smoothly from $\eta_{1, \infty}(T)$ to $\frac{h_{T}}{d} \eta_{1, \infty}(T)$ as $h_{T}+\operatorname{dist}(T, D)$ increases from $h_{T}$ to $O(d)$. The extra factor $h_{T}$ present in the latter error indicator significantly deemphasizes these contributions as the mesh is refined and, in particular, effectively measures the error contribution from these regions in $L_{\infty}$ instead of $W_{\infty}^{1}$.

The pollution term $C \frac{1}{d}\left(\left\|\mathbf{u}-\mathbf{u}_{h}\right\|_{L_{\infty}(\Omega)}+\max _{T \in \mathcal{T}_{h}} h_{T} \eta_{1, \infty}(T)\right)$ measures the influence of global solution properties on the local solution quality. Following [SL06], it can be bounded a posteriori, as indicated in the corollary below.

The regularization penalty $C \rho^{\beta}\left(|\mathbf{u}|_{C^{1, \beta}\left(\overline{B_{\rho}}\right)}+|p|_{C^{0, \beta}\left(\overline{B_{\rho}}\right)}\right)$ is due to technicalities associated with bounding maximum norms and, in particular, to regularization of the Green's function in our arguments. Because $D$ is away from reentrant corners, it should be expected that $(\mathbf{u}, p) \in C^{1, \beta}\left(\overline{B_{\rho}}\right) \times C^{0, \beta}\left(\overline{B_{\rho}}\right)$ for some $\beta \in(0,1)$. Following this observation, we use local Hölder regularity estimates in order to control the regularization penalty by data in the corollary below. However, the results available in the literature restrict our choice of the compressibility $g$ somewhat in the case $n=3$, though the incompressible case $g=0$ is always allowed. After obtaining local regularity bounds, we make use of our ability to choose $\rho$ arbitrarily small in order to obtain a final, completely a posteriori bound.

Note finally that we assume the validity of certain Green's function estimates in Theorem 1 because the precise estimates that we need are not quite available in 
the literature in the case $n=2$. The needed estimates have been published in the case $n=3$ and almost certainly hold as well for $n=2$; see the discussion following Lemma 10.

Corollary 2. Let the Green's functions estimates of Lemma 10 below for convex polyhedral domains hold. In the spatial dimension $n=3$ assume also that the incompressiblity $g$ in (1.1) is 0 in a neighborhood of all edges of $\Omega$ lying within a distance $d$ of $D$. Define

$$
\begin{aligned}
& F(\mathbf{f}, g, d)=\|\mathbf{f}\|_{L_{q}(\Omega)}+|g|_{W_{q}^{1}(\Omega)}+d^{-2}\left(\|\mathbf{f}\|_{W_{q}^{-1}(\Omega)}+\|g\|_{L_{q}(\Omega)}\right), \\
& \mathcal{E}=\max _{T \in \mathcal{T}_{D_{d}}} \frac{h_{T}}{h_{T}+\operatorname{dist}(T, D)} \eta_{1, \infty}(T), \\
& \beta=1-\frac{n}{q}, \quad \tilde{\rho}=\min \left(d, \underline{h},(\mathcal{E} / F(\mathbf{f}, g, d))^{1 / \beta}\right) .
\end{aligned}
$$

Then there exists $q>n$ sufficiently close to $n$ and depending on $\Omega$ and $D_{d}$ such that the following dichotomy holds.

If $\mathcal{E}=0$, then

$$
\left\|\nabla\left(\mathbf{u}-\mathbf{u}_{h}\right)\right\|_{L_{\infty}(D)}+\left\|p-p_{h}\right\|_{L_{\infty}(D)} \leq C\left(\ln \frac{1}{\underline{h}}\right)^{\alpha_{n}} \frac{1}{d} \max _{T \in \mathcal{T}_{h}} h_{T} \eta_{1, \infty}(T) .
$$

If $\mathcal{E} \neq 0$, then

$$
\begin{aligned}
\left\|\nabla\left(\mathbf{u}-\mathbf{u}_{h}\right)\right\|_{L_{\infty}(D)}+ & \left\|p-p_{h}\right\|_{L_{\infty}(D)} \\
\leq & C\left(1+\ln \frac{d}{\tilde{\rho}}\right) \mathcal{E}+C\left(\ln \frac{1}{\underline{h}}\right)^{\alpha_{n}} \frac{1}{d} \max _{T \in \mathcal{T}_{h}} h_{T} \eta_{1, \infty}(T) .
\end{aligned}
$$

Here $\alpha_{2}=2$ and $\alpha_{3}=4 / 3$.

Because we expect convergence to be faster in $L_{\infty}$ than in $W_{\infty}^{1}$, the pollution term in (1.8) is of higher order when viewed from the perspective of a priori convergence rates. Adaptive algorithms based on (1.8) can correspondingly be expected to generate coarser meshes in $\Omega \backslash D_{d}$ than in $D_{d}$.

The logarithmic factor $\ln d / \tilde{\rho}$ in (1.8) above is nonstandard, so we briefly remark on it. When $(\mathcal{E} / F)^{1 / \beta}<\underline{h}$, the logarithmic factor will depend on $\mathcal{E}$ and on $d$, but not on the meshsize $\underline{h}$. In the standard literature on maximum norm error estimates such logarithmic factors depend only on the (minimum) meshsize. In the typical case where $\mathcal{E} \geq C \underline{h}^{k}$, the possible presence of $\mathcal{E}$ does not change the essential nature of the logarithmic factor at all. Only in the exceptional case $\mathcal{E} \ll \underline{h}^{k}$ does the nature of the log factor become nonstandard. The presence of the computable estimator $\mathcal{E}$ in the logarithmic factor allows us to obtain a completely a posteriori bound.

We finally give a brief discussion of related literature. Local a priori error estimates for the Stokes equation are developed in [HXZL08 and used to justify a local parallel finite element algorithm. Global $W_{\infty}^{1}$ a priori error estimates for the Stokes system on convex polygonal and polyhedral domains can be found in the recent papers GNS04, GNS05, and GL10; we also refer to these works for a more comprehensive overview of previous literature on maximum norm a priori analysis for the Stokes problem.

As mentioned above, SL06 contains global a posteriori error estimates in the maximum norm for the Stokes equation. Local a posteriori estimates for maximum 
gradient errors for Poisson's problem are proved in Dem07]; related global maximum gradient error estimators are developed in Dem06. In both of these works, the regularization penalty in (1.6) was essentially assumed a priori to be small. Our treatment here is entirely a posteriori, so in this regard we obtain sharper results than have previously been available even for Poisson's problem.

An outline of the paper is as follows. In Section 2 we give several preliminary definitions. In Section 3 we use a duality argument in order to represent the local pointwise gradient error, and in Section 4 we complete the proof of Theorem 1 by proving a number of regularity estimates. In Section 5 we prove Corollary 2 , Finally, in Section 6 we present a computational example.

\section{Preliminaries}

2.1. Finite element spaces and interpolants. We employ the standard TaylorHood finite element spaces. Let $V_{h}$ be the continuous piecewise polynomials of degree $k$ and let $X_{h}$ be the continuous piecewise polynomials of degree $k-1, k \geq 2$. With this definition, existence of solutions to (1.4) is well known to hold, as well as uniqueness, so long as $\int_{\Omega} p_{h} \mathrm{~d} x=0$ is enforced.

We also assume the existence of interpolation operators $I_{h}: V \rightarrow V_{h}, J_{h}: X \rightarrow$ $X_{h}$ such that, for $1 \leq p \leq \infty$,

$$
\begin{aligned}
\left\|I_{h} v-v\right\|_{W_{p}^{j}(T)} & \leq C h_{T}^{m-j}|v|_{W_{p}^{m}\left(P_{T}\right)}, \quad j=0,1, m=1,2, \\
\left\|J_{h} p-p\right\|_{L_{p}(T)} & \leq C h_{T}^{m}|p|_{W_{p}^{m}\left(P_{T}\right)}, \quad m=0,1 .
\end{aligned}
$$

These are standard properties of interpolants of Clément or Scott-Zhang type. Also, $P_{T}$ is the patch of elements touching $T$, with a corresponding hierarchy of neighbor patches of a simplex $T \in \mathcal{T}_{h}$ defined by:

$$
\begin{aligned}
& P_{T}=\bigcup\left\{S \in \mathcal{T}_{h}: \bar{S} \cap \bar{T} \neq \emptyset\right\}, \\
& P_{T}^{\prime}=\bigcup\left\{S \in \mathcal{T}_{h}: \bar{S} \cap \bar{P}_{T} \neq \emptyset\right\}, \\
& P_{T}^{\prime \prime}=\bigcup\left\{S \in \mathcal{T}_{h}: \bar{S} \cap \bar{P}_{T}^{\prime} \neq \emptyset\right\}, \quad \text { etc. }
\end{aligned}
$$

We finally note that $I_{h}$ and $J_{h}$ may be defined so that, if $\mathbf{v} \in\left(H_{0}^{1}\left(D_{d}\right)\right)^{n}$ and $q \in L_{2}(\Omega)$ is supported in $D_{d}$, then $I_{h} \mathbf{v}$ and $J_{h} q$ have support in $\mathcal{T}_{D_{d}}$.

2.2. Reference domains. Our proofs involve carrying out duality arguments over subdomains $B$ of $\Omega$. To describe these we recall that the target domain $D$ is a fixed distance away from reentrant corners or edges but may touch the remaining parts of $\partial \Omega$. We fix a point $x_{0} \in D$ where the maximum error over $D$ is attained; see 3.1. When $\operatorname{dist}\left(x_{0}, \partial \Omega\right) \geq d$ we can choose our subdomain $B$ to be a square or cube with diameter $d$ centered at $x_{0}$. When $x_{0}$ is close to $\partial \Omega$ we must carefully control the size and shape of these subdomains in order to ensure that regularity constants appearing in our estimates are uniformly bounded. We thus define reference domains to which we may map portions of $\Omega$ lying near $\partial \Omega$. A similar approach was used in Dem07, and we refer to $\S 2.2$ of that work for more detail. In particular, it is shown there that there exists a set $\left\{\tilde{B}_{1}, \ldots, \tilde{B}_{M}\right\}$ of reference domains of unit diameter, each of which is a convex polyhedron, with the following properties.

There exists a constant $d_{0} \leq 1$ depending on $\Omega$ such that whenever $d \leq d_{0}$, the following hold: Assume that $x_{0} \in \Omega$ with $\operatorname{dist}\left(x_{0}, e\right) \geq d$ for all reentrant corners or edges $e$ of $\partial \Omega$. Then there exist constants $c_{1}>0$ and $0<c_{2} \leq 1$, independent of 
$x_{0}$, and a subdomain $B$ of $\Omega$ such that $x_{0} \in B$, $\operatorname{dist}\left(x_{0}, \partial B \backslash \partial \Omega\right) \geq c_{1} d$, and such that for some $1 \leq i \leq M$ there is an affine bijection $A_{i}: \tilde{B}_{i} \rightarrow B$, where $A_{i}$ consists only of translation and scaling by $c d$ for some $c_{2} \leq c \leq 1$.

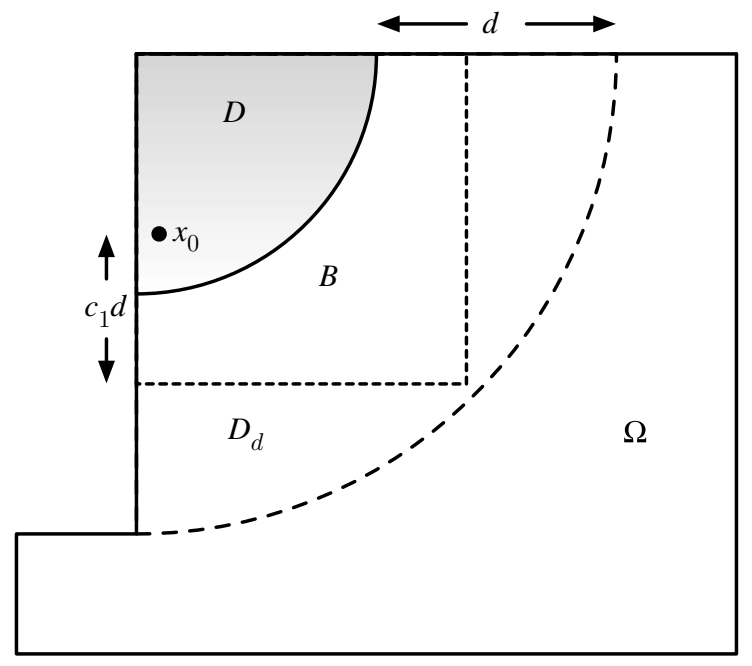

Figure 1. Diagram indicating relative positions of $\Omega, D$ (shaded), $D_{d}$ (with dashed boundary), and the reference domain $B$ (a square with fine dashed boundary). In this case the unit reference domain $\tilde{B}$ would be a unit square.

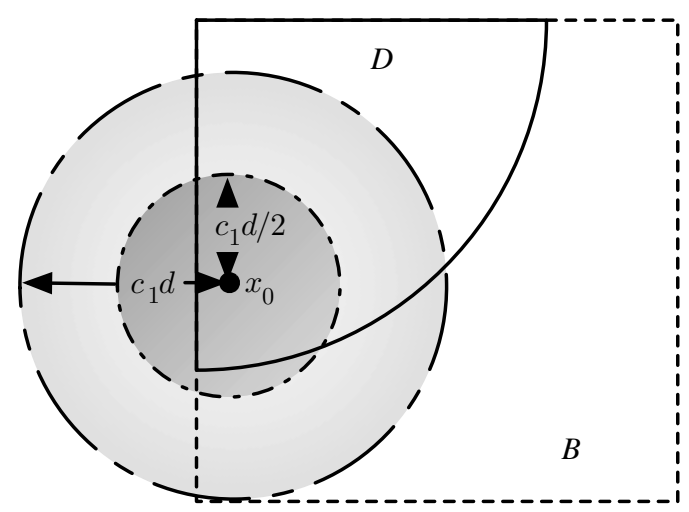

Figure 2. Blowup of $B$, showing $x_{0}, B_{c_{1} d / 2}\left(x_{0}\right)$ (darker shaded disc), and $B_{c_{1} d}\left(x_{0}\right)$ (lightly shaded disc). $B_{d / 2}$ is defined as the connected component containing $x_{0}$ of the intersection of $B_{c_{1} d / 2}\left(x_{0}\right)$ with $\Omega$.

We will also use a cut-off function $\omega$. With $x_{0}$ and $c_{1}$ as above, let $\omega \in$ $C_{0}^{\infty}\left(B_{c_{1} d}\left(x_{0}\right)\right)$ satisfy

$$
\omega \equiv 1 \text { on } B_{c_{1} d / 2}\left(x_{0}\right)
$$


Here $B_{r}(x)$ denotes the open ball with radius $r$ and center $x$. If $B_{c_{1} d}\left(x_{0}\right) \cap \Omega$ is not connected, then we assume that $\omega \equiv 0$ on any component of $B_{c_{1} d}\left(x_{0}\right) \cap \Omega$ not containing $x_{0}$. Note that $\omega=0$ on $\partial B \backslash \partial \Omega$ and that $\omega$ may be defined so that

$$
\|\omega\|_{W_{\infty}^{j}(B)} \leq C_{j} d^{-j}, \quad j=0,1,2, \ldots .
$$

In summary, $D$ is the "target" domain for error control, while $D_{d}$ is a larger "cut-off" domain with cut-off parameter $d$. Because we cannot control the shape of $D$ and $D_{d}$ and attendant regularity constants, we also define reference domains $B \subset D_{d}$ which has one of a finite number of pre-defined polygonal shapes and which may be obtained essentially by scaling a reference domain by $d$. Finally, $B_{c_{1}} d\left(x_{0}\right)$ is the support of the cut-off function $\omega$ which will play an essential role in our arguments. See Figure 1 and Figure 2 for a visual representation of these definitions.

\subsection{A posteriori estimates in $L_{\infty}$. We quote a result from [SL06].}

Lemma 3. Assume that $\Omega$ is a polyhedral domain in $\mathbb{R}^{n}, n=2,3$. Then

$$
\left\|\mathbf{u}-\mathbf{u}_{h}\right\|_{L_{\infty}(\Omega)} \leq C\left(\ln \frac{1}{\underline{h}}\right)^{\alpha_{n}} \max _{T \in \mathcal{T}_{h}} h_{T} \eta_{1, \infty}(T) .
$$

Here $\alpha_{2}=2$ and $\alpha_{3}=4 / 3$.

Remark 4. The arguments of [SL06] and ours below are valid for polyhedral domains with Lipschitz boundary, which excludes crack domains and other non-Lipschitz polyhedra. However, similar estimates for Poisson's problem can also be obtained for polyhedral domains such as crack domains that do not have Lipschitz boundaries (cf. DG10]), and it is likely that the same is true for the Stokes system. The reason for this difference is that certain relevant regularity estimates for the Stokes system are only available in the literature for Lipschitz domains. Our numerical examples are carried out on a two-dimensional domain with a crack.

\section{ERROR REPRESENTATION}

In this section we represent $\left\|\nabla\left(\mathbf{u}-\mathbf{u}_{h}\right)\right\|_{L_{\infty}(D)}$ and $\left\|p_{h}-p\right\|_{L_{\infty}(D)}$ by employing a regularized Green's function.

\subsection{Pointwise pressure and velocity gradient errors. Let}

$$
\mathbf{e}_{\mathbf{u}}=\mathbf{u}_{h}-\mathbf{u}, \quad e_{p}=p_{h}-p .
$$

Our goal is to simultaneously bound $\left\|\nabla \mathbf{e}_{\mathbf{u}}\right\|_{L_{\infty}(D)}$ and $\left\|e_{p}\right\|_{L_{\infty}(D)}$. We begin by selecting $x_{0} \in \bar{D}$ and $i, j$ so that

$$
\begin{aligned}
\sup _{x \in D}\left(\max _{k, l}\left|D_{k} \mathbf{e}_{\mathbf{u} l}(x)\right|+\left|e_{p}(x)\right|\right) & =\left|D_{i} \mathbf{e}_{\mathbf{u} j}\left(x_{0}\right)\right|+\left|e_{p}\left(x_{0}\right)\right| \\
& =\left|D_{i} \mathbf{e}_{\mathbf{u}}\left(x_{0}\right) \cdot \mathbf{k}_{j}\right|+\left|e_{p}\left(x_{0}\right)\right| .
\end{aligned}
$$

Here $\mathbf{k}_{j}$ denotes the $j$-th basis vector. Making the definition $\left\|\nabla \mathbf{e}_{\mathbf{u}}\right\|_{L_{\infty}(D)}=$ $\sup _{x \in D} \max _{k, l}\left|D_{k} \mathbf{e}_{\mathbf{u} l}(x)\right|$, we see that $\left\|\nabla \mathbf{e}_{\mathbf{u}}\right\|_{L_{\infty}(D)}+\left\|e_{p}\right\|_{L_{\infty}(D)}$ is bounded up to a factor two by the above expression.

We shall express the above errors by means of an approximate "delta function" at $x_{0}: D_{i} \mathbf{e}_{\mathbf{u}}\left(x_{0}\right) \cdot \mathbf{k}_{j} \approx\left(D_{i} \mathbf{e}_{\mathbf{u}} \cdot \mathbf{k}_{j}, \delta\right)=\left(D_{i} \mathbf{e}_{\mathbf{u}}, \delta \mathbf{k}_{j}\right)$ and $e_{p}\left(x_{0}\right) \approx\left(e_{p}, \delta\right)$. In order to do so, we select a simplex $T_{x_{0}} \in \mathcal{T}_{h}$ such that

$$
x_{0} \in \overline{T_{x_{0}}} \text {. }
$$


Furthermore, we define a regularized "delta function" $\delta$ corresponding to the point $x_{0}$ (cf. SW95]). Following $\S 2.3$ of [Dem07, we let $\rho$ be as in Theorem 1 and fix a shape-regular simplex $T_{0}$ of diameter $\rho$ such that $x_{0} \in T_{0} \subset T_{x_{0}}$; see Figure 3 . More precisely, $T_{0}$ should satisfy the same regularity assumption as the simplices in the mesh family $\left\{\mathcal{T}_{h}\right\}$. Then $\delta \in C_{0}^{\infty}\left(T_{0}\right)$ may be defined so that for any polynomial $P$ of degree at most $k-1$ (where $k$ is the polynomial degree of $V_{h}$ ),

$$
\begin{aligned}
& P\left(x_{0}\right)=\int_{T_{0}} \delta P \mathrm{~d} x, \\
& \|\delta\|_{W_{p}^{j}\left(T_{0}\right)} \leq C \rho^{-j-n\left(1-\frac{1}{p}\right)}, \quad 1 \leq p \leq \infty, j=0,1 .
\end{aligned}
$$

Following precisely the arguments in Proposition 2.3 of [Dem07, we obtain the following.

Lemma 5. Assume that $(\mathbf{u}, p) \in C^{1, \beta}\left(\overline{T_{0}}\right) \times C^{0, \beta}\left(\overline{T_{0}}\right)$ for some $\beta \in(0,1)$ and that $\left(\mathbf{v}_{h}, q_{h}\right) \in V_{h} \times X_{h}$. Then

$$
\begin{aligned}
\left|D_{i}\left(\mathbf{u}_{j}-\mathbf{v}_{h j}\right)\left(x_{0}\right)\right|+\left|p\left(x_{0}\right)-q_{h}\left(x_{0}\right)\right| \leq & \left|\left(D_{i}\left(\mathbf{u}_{j}-\mathbf{v}_{h j}\right), \delta\right) \pm\left(p-q_{h}, \delta\right)\right| \\
& +C \rho^{\beta}\left(|\mathbf{u}|_{C^{1, \beta}\left(\overline{T_{0}}\right)}+|p|_{C^{0, \beta}\left(\overline{T_{0}}\right)}\right) .
\end{aligned}
$$

Here the sign of $\pm\left(p-q_{h}, \delta\right)$ is chosen to match the sign of $\left(D_{i}\left(\mathbf{u}_{j}-\mathbf{v}_{h j}\right), \delta\right)$.

Employing (3.2), we obtain the following error representation.

Lemma 6. Under the assumptions of Theorem 1 we have

$$
\begin{aligned}
\left\|\nabla \mathbf{e}_{\mathbf{u}}\right\|_{L_{\infty}(D)}+\left\|e_{p}\right\|_{L_{\infty}(D) \leq} & 2\left|\left(D_{i} \mathbf{e}_{\mathbf{u}}, \delta \mathbf{k}_{j}\right) \pm\left(e_{p}, \delta\right)\right| \\
& +C \rho^{\beta}\left(|\mathbf{u}|_{C^{1, \beta}\left(\overline{B_{\rho}}\right)}+|p|_{C^{0, \beta}\left(\overline{B_{\rho}}\right)}\right),
\end{aligned}
$$

where as above the sign of $\pm\left(e_{p}, \delta\right)$ is chosen to match the sign of $\left(D_{i} \mathbf{e}_{\mathbf{u}}, \delta \mathbf{k}_{j}\right)$.
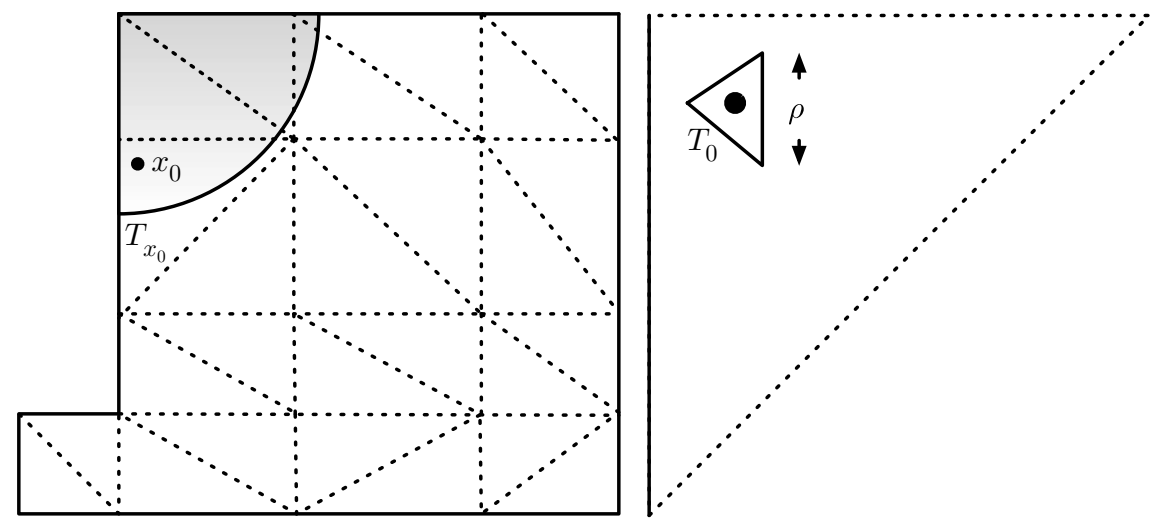

FiguRE 3. Left: Triangulated domain $\Omega$ with $D$ indicated (shaded area) and $x_{0}$ and $T_{x_{0}}$ labeled. Right: Blowup of $T_{x_{0}}$ with $x_{0}$ indicated (dot) and $T_{0}$ labeled. 
3.2. Localization. As above, we let $d$ be the distance from the target subdomain $D$ to the nearest reentrant edge or vertex in $\partial \Omega$. Let $x_{0} \in D$ be a point where the maximum error is attained and choose the subdomain $B$ and the cut-off function $\omega$ as in $\$ 2.2$. As in Theorem 1 we assume that $\rho \leq c_{0} d$ for $c_{0}$ sufficiently small. Hence, we may achieve $\omega \equiv 1$ on $\operatorname{supp}(\delta)$ and $\operatorname{supp}(\delta) \subset T_{0} \subset B$, so that the main term in (3.3) becomes

$$
\left(D_{i} \mathbf{e}_{\mathbf{u}}, \delta \mathbf{k}_{j}\right)-\left(e_{p}, \delta\right)=-\left(\mathbf{e}_{\mathbf{u}}, D_{i} \delta \mathbf{k}_{j}\right)-\left(e_{p}, \delta\right)=-\left(\omega \mathbf{e}_{\mathbf{u}}, D_{i} \delta \mathbf{k}_{j}\right)-\left(\omega e_{p}, \delta\right) .
$$

For the sake of notational simplicity we assumed here that the sign in front of $\left(e_{p}, \delta\right)$ is -; the actual sign has no effect on our arguments below. We additionally let $\delta_{B}$ be a smooth function satisfying $\int_{B}\left(\delta-\delta_{B}\right) \mathrm{d} x=0$; a precise definition will be made in $\$ 4.2$ below. We introduce a localized adjoint problem: find $(\mathbf{v}, q) \in V_{B} \times X_{B}$ with $\int_{B} q \mathrm{~d} x=0$ such that

$$
\mathcal{L}_{B}((\mathbf{w}, \lambda),(\mathbf{v}, q))=\left(\mathbf{w}, D_{i} \delta \mathbf{k}_{j}\right)+\left(\lambda, \delta-\delta_{B}\right) \quad \forall(\mathbf{w}, \lambda) \in V_{B} \times X_{B},
$$

where the form $\mathcal{L}_{B}$ is defined as in (1.2) 1.3 but with integrals extending only over $B$ and $V_{B} \times X_{B}=\left(H_{0}^{1}(B)\right)^{n} \times L_{2}(B)$. The strong form is:

$$
\begin{aligned}
-\Delta \mathbf{v}-\nabla q & =D_{i} \delta \mathbf{k}_{j}, & & \text { in } B, \\
-\nabla \cdot \mathbf{v} & =\delta-\delta_{B}, & & \text { in } B, \\
\mathbf{v} & =0, & & \text { on } \partial B .
\end{aligned}
$$

We extend $\mathbf{v}$ by zero outside of $B$. Note that $\omega \mathbf{e}_{\mathbf{u}} \in V_{B}$ because $\omega=0$ on $\partial B \backslash \partial \Omega$ (since $\left.\operatorname{dist}\left(x_{0}, \partial B \backslash \partial \Omega\right) \geq c_{1} d\right)$ and $\mathbf{e}_{\mathbf{u}}=0$ on $\partial \Omega$.

3.3. Duality argument. We choose $(\mathbf{w}, \lambda)=\left(\omega \mathbf{e}_{\mathbf{u}}, \omega e_{p}\right) \in V_{B} \times X_{B}$ in (3.5), use (3.4), and recall that $\mathbf{v}=0$ outside $B$. Thus,

$$
\begin{aligned}
-\left(D_{i} \mathbf{e}_{\mathbf{u}}, \delta \mathbf{k}_{j}\right)+\left(e_{p}, \delta\right) & =\left(\omega \mathbf{e}_{\mathbf{u}}, D_{i} \delta \mathbf{k}_{j}\right)+\left(\omega e_{p}, \delta-\delta_{B}\right)+\left(\omega e_{p}, \delta_{B}\right) \\
& =\mathcal{L}_{B}\left(\left(\omega \mathbf{e}_{\mathbf{u}}, \omega e_{p}\right),(\mathbf{v}, q)\right)+\left(e_{p}, \omega \delta_{B}\right) \\
& =a\left(\omega \mathbf{e}_{\mathbf{u}}, \mathbf{v}\right)+b\left(\mathbf{v}, \omega e_{p}\right)-b\left(\omega \mathbf{e}_{\mathbf{u}}, q\right)+\left(e_{p}, \omega \delta_{B}\right) .
\end{aligned}
$$

Writing $a(\mathbf{u}, \mathbf{v})=\int_{\Omega} \mathbf{u}_{i, j} \mathbf{v}_{i, j} \mathrm{~d} x$, performing elementary manipulations, and integrating by parts yields

$$
\begin{aligned}
a\left(\omega \mathbf{e}_{\mathbf{u}}, \mathbf{v}\right) & =\int_{\Omega}\left[\omega \mathbf{e}_{\mathbf{u} i, j} \mathbf{v}_{i, j}+\mathbf{e}_{\mathbf{u} i} \omega_{, j} \mathbf{v}_{i, j}\right] \mathrm{d} x \\
& =\int_{\Omega}\left[\mathbf{e}_{\mathbf{u} i, j}(\omega \mathbf{v})_{i, j}-\mathbf{e}_{\mathbf{u} i, j} \omega_{, j} \mathbf{v}_{i}+\mathbf{e}_{\mathbf{u} i} \omega_{, j} \mathbf{v}_{i, j}\right] \mathrm{d} x \\
& =a\left(\mathbf{e}_{\mathbf{u}}, \omega \mathbf{v}\right)+\int_{\Omega}\left[\mathbf{e}_{\mathbf{u} i}\left(\omega_{, j} \mathbf{v}_{i}\right)_{, j}+\mathbf{e}_{\mathbf{u} i} \omega_{, j} \mathbf{v}_{i, j}\right] \mathrm{d} x \\
& =a\left(\mathbf{e}_{\mathbf{u}}, \omega \mathbf{v}\right)+\int_{\Omega}\left[\mathbf{e}_{\mathbf{u} i} v_{i} \omega_{, j j}+2 \mathbf{e}_{\mathbf{u} i} \omega_{, j} \mathbf{v}_{i, j}\right] \mathrm{d} x \\
& =a\left(\mathbf{e}_{\mathbf{u}}, \omega \mathbf{v}\right)+\left(\mathbf{e}_{\mathbf{u}}, \mathbf{v} \Delta \omega+2 \nabla \mathbf{v} \nabla \omega\right) .
\end{aligned}
$$

Similarly,

$$
\begin{aligned}
b\left(\mathbf{v}, \omega e_{p}\right)-b\left(\omega \mathbf{e}_{\mathbf{u}}, q\right) & =-\left(\nabla \cdot \mathbf{v}, \omega e_{p}\right)+\left(\nabla \cdot\left(\omega \mathbf{e}_{\mathbf{u}}\right), q\right) \\
& =-\left(\nabla \cdot(\omega \mathbf{v}), e_{p}\right)+\left(\mathbf{v} \cdot \nabla \omega, e_{p}\right)+\left(\nabla \omega \cdot \mathbf{e}_{\mathbf{u}}, q\right)+\left(\nabla \cdot \mathbf{e}_{\mathbf{u}}, \omega q\right) \\
& =b\left(\omega \mathbf{v}, e_{p}\right)-b\left(\mathbf{e}_{\mathbf{u}}, \omega q\right)+\left(\mathbf{v} \cdot \nabla \omega, e_{p}\right)+\left(\mathbf{e}_{\mathbf{u}}, q \nabla \omega\right) .
\end{aligned}
$$


Therefore, (3.6) can be written

$$
\begin{aligned}
-\left(D_{i} \mathbf{e}_{\mathbf{u}}, \delta \mathbf{k}_{j}\right)+\left(e_{p}, \delta\right)= & \mathcal{L}\left(\left(\mathbf{e}_{\mathbf{u}}, e_{p}\right),(\omega \mathbf{v}, \omega q)\right)+\left(e_{p}, \omega \delta_{B}+\mathbf{v} \cdot \nabla \omega\right) \\
& +\left(\mathbf{e}_{\mathbf{u}}, \mathbf{v} \Delta \omega+2 \nabla \mathbf{v} \nabla \omega+q \nabla \omega\right) .
\end{aligned}
$$

Setting $B_{d / 2}=B_{c_{1} d / 2}\left(x_{0}\right) \cap B$, recalling from (2.3) that $\omega \equiv 1$ and thus $\nabla \omega \equiv 0$ on $B_{d / 2}$, and recalling from (2.4) that $\left.\mid D^{k} \omega\right) \mid \leq C d^{-k}$, we conclude that

$$
\begin{aligned}
& \left|\left(D_{i} \mathbf{e}_{\mathbf{u}}, \delta \mathbf{k}_{j}\right)-\left(e_{p}, \delta\right)\right| \leq\left|\mathcal{L}\left(\left(\mathbf{e}_{\mathbf{u}}, e_{p}\right),(\omega \mathbf{v}, \omega q)\right)\right|+\left|\left(e_{p}, \omega \delta_{B}+\mathbf{v} \cdot \nabla \omega\right)\right| \\
& +C d^{-1}\left\|\mathbf{e}_{\mathbf{u}}\right\|_{L_{\infty}(B)}\left(d^{-1}\|\mathbf{v}\|_{L_{1}\left(B \backslash B_{d / 2}\right)}+\|\nabla \mathbf{v}\|_{L_{1}\left(B \backslash B_{d / 2}\right)}+\|q\|_{L_{1}\left(B \backslash B_{d / 2}\right)}\right) .
\end{aligned}
$$

We now consider the term $\left|\mathcal{L}\left(\left(\mathbf{e}_{\mathbf{u}}, e_{p}\right),(\omega \mathbf{v}, \omega q)\right)\right|$. Galerkin orthogonality implies

$$
\mathcal{L}\left(\left(\mathbf{e}_{\mathbf{u}}, e_{p}\right),(\omega \mathbf{v}, \omega q)\right)=\mathcal{L}\left(\left(\mathbf{e}_{\mathbf{u}}, e_{p}\right),\left(\omega \mathbf{v}-I_{h}(\omega \mathbf{v}), \omega q-J_{h}(\omega q)\right)\right) .
$$

Recalling that $\operatorname{supp}\left(\omega \mathbf{v}-I_{h}(\omega \mathbf{v})\right) \subset \mathcal{T}_{D_{d}}$ and employing standard techniques for proving residual error estimates (cf. Dem07] for similar computations), we next compute

$$
\begin{aligned}
& \mid \mathcal{L}\left(\left(\mathbf{e}_{\mathbf{u}}, e_{p}\right),\left(\omega \mathbf{v}-I_{h}(\omega \mathbf{v}), \omega q-J_{h}(\omega q)\right) \mid\right. \\
& \leq C \sum_{T \in \mathcal{T}_{D_{d}}} \eta_{1, \infty}(T)\left(h_{T}^{-1}\left\|\omega \mathbf{v}-I_{h}(\omega \mathbf{v})\right\|_{L_{1}(T)}\right. \\
& \left.\quad+\left\|\nabla\left(\omega \mathbf{v}-I_{h}(\omega \mathbf{v})\right)\right\|_{L_{1}(T)}+\left\|\omega q-J_{h}(\omega q)\right\|_{L_{1}(T)}\right) \\
& \leq C \max _{T \in \mathcal{T}_{D_{d}}} \frac{h_{T}}{h_{T}+\operatorname{dist}(T, D)} \eta_{1, \infty}(T) \\
& \quad \times \sum_{T \in \mathcal{T}_{D_{d}}} \frac{h_{T}+\operatorname{dist}\left(T, T_{x_{0}}\right)}{h_{T}}\left(h_{T}^{-1}\left\|\omega \mathbf{v}-I_{h}(\omega \mathbf{v})\right\|_{L_{1}(T)}\right. \\
& \left.\quad+\left\|\nabla\left(\omega \mathbf{v}-I_{h}(\omega \mathbf{v})\right)\right\|_{L_{1}(T)}+\left\|\omega q-J_{h}(\omega q)\right\|_{L_{1}(T)}\right) .
\end{aligned}
$$

Here we used that $h_{T}+\operatorname{dist}\left(T, T_{x_{0}}\right) \geq C\left(h_{T}+\operatorname{dist}(T, D)\right)$, so that $\frac{h_{T}}{h_{T}+\operatorname{dist}\left(T, T_{x_{0}}\right)} \leq$ $C_{\frac{h_{T}}{h_{T}+\operatorname{dist}(T, D)}}$.

Recall that we assume $\rho \leq c_{0} \underline{h}$. We choose $c_{0}$ so small that $B_{2 \rho}\left(x_{0}\right) \cap \Omega \subset P_{T_{x_{0}}}^{\prime}$ and then apply the interpolation estimates (2.1) and (2.2) to the terms in (3.10) as follows. Recall that $x_{0} \in T_{x_{0}}$. For $T \in P_{T_{x_{0}}}^{\prime \prime}$ we apply (2.1) with $m=1$ and (2.2) with $m=0$, while for all other $T \in \mathcal{T}_{D_{d}}$ we use $m=2$ and $m=1$, respectively. We also note that $h_{T}+\operatorname{dist}\left(T, T_{x_{0}}\right) \simeq h_{T}$ for $T \in P_{T_{x_{0}}}^{\prime \prime}$ and that $h_{T}+\operatorname{dist}\left(T, T_{x_{0}}\right) \simeq \operatorname{dist}\left(x, x_{0}\right)$ for $x \in T \in \mathcal{T}_{D_{d}} \backslash P_{T_{x_{0}}}^{\prime}$.

More precisely, let

$$
\begin{aligned}
I(T)= & \frac{h_{T}+\operatorname{dist}\left(T, T_{x_{0}}\right)}{h_{T}}\left(h_{T}^{-1}\left\|\omega \mathbf{v}-I_{h}(\omega \mathbf{v})\right\|_{L_{1}(T)}\right. \\
& \left.+\left\|\nabla\left(\omega \mathbf{v}-I_{h}(\omega \mathbf{v})\right)\right\|_{L_{1}(T)}+\left\|\omega q-J_{h}(\omega q)\right\|_{L_{1}(T)}\right) .
\end{aligned}
$$

When bounding $\sum_{T \in \mathcal{T}_{D_{d}}} I(T)$ in (3.10), we have for the terms with $T \in P_{T_{x_{0}}}^{\prime \prime}$

$$
\begin{aligned}
\sum_{T \in P_{T_{x_{0}}}^{\prime \prime}} I(T) & \leq C \sum_{T \in P_{T_{x_{0}}}^{\prime \prime}}\left(\|\nabla(\omega \mathbf{v})\|_{L_{1}\left(P_{T}\right)}+\|\omega q\|_{L_{1}\left(P_{T}\right)}\right) \\
& \leq C\left(d^{-1}\|\mathbf{v}\|_{L_{1}\left(P_{T_{x_{0}}}^{\prime \prime \prime}\right)}+\|\nabla \mathbf{v}\|_{L_{1}\left(P_{T_{x_{0}}}^{\prime \prime \prime}\right)}+\|q\|_{L_{1}\left(P_{T_{x_{0}}}^{\prime \prime \prime}\right)}\right) .
\end{aligned}
$$


For the terms with $T \notin P_{T_{x_{0}}}^{\prime \prime}$, we first note that for $T$ with $T \cap(\partial B \backslash \partial \Omega) \neq \emptyset, \mathbf{v} \notin$ $W_{1}^{2}(T)$ since $\partial B$ may cut through the interior of $T$, but we do have $\omega \mathbf{v} \in W_{1}^{2}(T)$. We then use $B_{2 \rho}\left(x_{0}\right) \subset P_{T_{x_{0}}}^{\prime}$, (2.4), $\left|x-x_{0}\right| \leq d$ for all $x \in B$, the scaled Poincaré inequality $\|\mathbf{v}\|_{L_{1}(B)} \leq C d\|\nabla \mathbf{v}\|_{L_{1}(B)}$, and that $\omega \mathbf{v}, \omega q$ vanish outside $B$ to obtain

$$
\begin{aligned}
& \sum_{T \notin P_{T_{x_{0}}}^{\prime \prime}} I(T) \leq C \sum_{T \notin P_{T_{x_{0}}^{\prime \prime}}}\left(h_{T}+\operatorname{dist}\left(T, T_{x_{0}}\right)\right)\left(\left\|D^{2}(\omega \mathbf{v})\right\|_{L_{1}\left(P_{T}\right)}+\|\nabla(\omega q)\|_{L_{1}\left(P_{T}\right)}\right) \\
& \leq C \int_{\mathcal{T}_{D_{d}} \backslash P_{T_{x_{0}}}^{\prime}}\left|x-x_{0}\right|\left[|\omega|\left(|\nabla q|+\left|D^{2} \mathbf{v}\right|\right)+|\nabla \omega|(|q|+|\nabla \mathbf{v}|)+|\Delta \omega \| \mathbf{v}|\right] \mathrm{d} x \\
& \leq C\left(\|q\|_{L_{1}(B)}+\|\nabla \mathbf{v}\|_{L_{1}(B)}+d^{-1}\|\mathbf{v}\|_{L_{1}(B)}\right) \\
&+C \int_{B \backslash B_{2 \rho}\left(x_{0}\right)}\left|x-x_{0}\right|\left(|\nabla q|+\left|D^{2} \mathbf{v}\right|\right) \mathrm{d} x \\
& \leq C\left(\|q\|_{L_{1}(B)}+\|\nabla \mathbf{v}\|_{L_{1}(B)}\right)+C \int_{B \backslash B_{2 \rho}\left(x_{0}\right)}\left|x-x_{0}\right|\left(|\nabla q|+\left|D^{2} \mathbf{v}\right|\right) \mathrm{d} x
\end{aligned}
$$

Collecting the previous two inequalities while again applying a scaled Poincaré inequality, we obtain

$$
\begin{aligned}
\sum_{T \in \mathcal{T}_{D_{d}}} I(T) \leq & C\left(\|\nabla \mathbf{v}\|_{L_{1}(B)}+\|q\|_{L_{1}(B)}\right. \\
& \left.+\int_{B \backslash B_{2 \rho}\left(x_{0}\right)}\left|x-x_{0}\right|\left(|\nabla q|+\left|D^{2} \mathbf{v}\right|\right) \mathrm{d} x\right) .
\end{aligned}
$$

Collecting the previous results, we obtain the following error representation.

Lemma 7. Under the assumptions of Theorem 1 we have

$$
\begin{aligned}
& \left\|\nabla \mathbf{e}_{\mathbf{u}}\right\|_{L_{\infty}(D)}+\left\|e_{p}\right\|_{L_{\infty}(D)} \leq C \max _{T \in \mathcal{T}_{D_{d}}} \frac{h_{T}}{h_{T}+\operatorname{dist}(T, D)} \eta_{1, \infty}(T) \\
& \quad \times\left(\|\mathbf{v}\|_{W_{1}^{1}(B)}+\|q\|_{L_{1}(B)}+\int_{B \backslash B_{2 \rho\left(x_{0}\right)}}\left|x-x_{0}\right|\left(|\nabla q|+\left|D^{2} \mathbf{v}\right|\right) d x\right) \\
& +C d^{-1}\left\|\mathbf{e}_{\mathbf{u}}\right\|_{L_{\infty}(B)}\left(d^{-1}\|\mathbf{v}\|_{L_{1}\left(B \backslash B_{d / 2}\right)}+\|\nabla \mathbf{v}\|_{L_{1}\left(B \backslash B_{d / 2}\right)}+\|q\|_{L_{1}\left(B \backslash B_{d / 2}\right)}\right) \\
& +\left|\left(e_{p}, \omega \delta_{B}+\mathbf{v} \cdot \nabla \omega\right)\right|+C \rho^{\beta}\left(|\mathbf{u}|_{C^{1, \beta}\left(\overline{B_{\rho}}\right)}+|p|_{C^{0, \beta}\left(\overline{B_{\rho}}\right)}\right) .
\end{aligned}
$$

Proof. We collect (3.11) into (3.10) and subsequently into (3.9) and (3.8). Combining the result with (3.3) completes the proof.

3.4. Regularity estimates. The estimates contained in the following two lemmas compose the remainder of the proof of Theorem 11. The proof of these estimates is carried out in 4 . We remind the reader that $B_{d / 2}=B_{c_{1} d / 2}\left(x_{0}\right) \cap B$; see (2.3).

Lemma 8. Let $(\mathbf{v}, q) \in V_{B} \times X_{B}$ be the solution of (3.5). Then

$$
\begin{aligned}
\|\mathbf{v}\|_{W_{1}^{1}(B)}+\|q\|_{L_{1}(B)} & \leq C \ln \frac{d}{\rho}, \\
d^{-1}\|\mathbf{v}\|_{L_{1}\left(B \backslash B_{d / 2}\right)}+\|\nabla \mathbf{v}\|_{L_{1}\left(B \backslash B_{d / 2}\right)}+\|q\|_{L_{1}\left(B \backslash B_{d / 2}\right)} & \leq C, \\
\int_{B \backslash B_{2 \rho}\left(x_{0}\right)}\left|x-x_{0}\right|\left(|\nabla q(x)|+\left|D^{2} \mathbf{v}(x)\right|\right) d x & \leq C \ln \frac{d}{\rho} .
\end{aligned}
$$


In the next lemma we provide a computable bound for the term $\left|\left(e_{p}, \omega \delta_{B}+\mathbf{v} \cdot \nabla \omega\right)\right|$ in Lemma 7.

Lemma 9. Let $\mathbf{v}, q$, and $p$ be as above. Then

$$
\left|\left(e_{p}, \omega \delta_{B}+\mathbf{v} \cdot \nabla \omega\right)\right| \leq C \frac{1}{d} \max _{T \in \mathcal{T}_{h}} h_{T} \eta_{1, \infty}(T)
$$

\section{Regularity estimates}

In this section we prove Lemma 8 and Lemma 9 , First we collect some basic regularity results and properties of the Green's function for Stokes' problem from the literature and then use them to analyze regularized Green's functions.

4.1. Regularity and Green's matrix estimates for the Stokes system on convex polyhedral domains. We begin by stating a standard regularity result. Let $\tilde{B}=\tilde{B}_{i}$ be one of the unit reference domains $\tilde{B}_{i}$ defined in 2.2 Assume that $(\tilde{\mathbf{v}}, \tilde{q}) \in V_{\tilde{B}} \times X_{\tilde{B}}$ solves the adjoint problem

$$
\mathcal{L}_{\tilde{B}}((\mathbf{w}, \lambda),(\tilde{\mathbf{v}}, \tilde{q}))=(\mathbf{w}, \tilde{\mathbf{f}})_{\tilde{B}}+(\lambda, \tilde{g})_{\tilde{B}} \quad \forall(\mathbf{w}, \lambda) \in V_{\tilde{B}} \times X_{\tilde{B}},
$$

where $\tilde{\mathbf{f}} \in\left(H^{-1}(\tilde{B})\right)^{n}$ and $\tilde{g} \in X_{\tilde{B}} / \mathbb{R}$. Then

$$
\|\tilde{\mathbf{v}}\|_{H_{0}^{1}(\tilde{B})}+\|\tilde{q}\|_{L_{2}(\tilde{B})} \leq C\left(\|\tilde{\mathbf{f}}\|_{H^{-1}(\tilde{B})}+\|\tilde{g}\|_{L_{2}(\tilde{B})}\right) .
$$

Since $\tilde{B}$ is convex, we also have (cf. [MR07])

$$
\|\tilde{\mathbf{v}}\|_{H^{2}(\tilde{B})}+\|\tilde{q}\|_{H^{1}(\tilde{B})} \leq C\left(\|\tilde{\mathbf{f}}\|_{L_{2}(\tilde{B})}+\|\tilde{g}\|_{H^{1}(\tilde{B})}\right) .
$$

As normally stated in the literature, the above estimates require that $\int_{\tilde{B}} \tilde{q} \mathrm{~d} \tilde{x}=0$, but we modify this assumption slightly so that we can employ properties of Green's function for convex polyhedral domains from [MR10, p. 555] and the following. We let $\phi \in C_{0}^{\infty}(\tilde{B})$ with $\int_{\tilde{B}} \phi \mathrm{d} \tilde{x}=1$. We then require that $\int_{\tilde{B}} \tilde{q} \phi \mathrm{d} \tilde{x}=0$. Because $C_{0}^{\infty}$ is dense in $L_{2}(\tilde{B})$, we may for any given $\epsilon>0$ choose $\phi$ so that $\left\||\tilde{B}|^{-1}-\phi\right\|_{L_{2}(\tilde{B})} \leq \epsilon$. By choosing $\epsilon$ sufficiently small ( $\epsilon=\frac{1}{2}$ will suffice), we easily see that then (4.2) and (4.3) hold also for $\tilde{q}$ satisfying $\int_{\tilde{B}} \tilde{q} \phi \mathrm{d} \tilde{x}=0$ instead of the usual $\int_{\tilde{B}} \tilde{q} \mathrm{~d} \tilde{x}=0$. In what follows we take $\phi$ to be a fixed function that is sufficiently close to $|\tilde{B}|^{-1}$ in $L_{r}(\tilde{B})$ for a sufficiently large (but fixed and finite) range of values of $r$.

Next we state results for the Green's matrix for the adjoint problem (4.1).

Lemma 10. Assume that $\tilde{B} \subset \mathbb{R}^{n}, n=2,3$, is a convex polyhedral domain and let $(\tilde{\mathbf{v}}, \tilde{q})$ be the solution of (4.1). There exists $\left\{G_{l j}(\tilde{x}, \tilde{\xi})\right\}_{1 \leq l, j \leq n+1},(\tilde{x}, \tilde{\xi}) \in \tilde{B} \times \tilde{B}$, such that for $\tilde{x} \in \tilde{B}$ and $1 \leq l \leq n$,

$$
\begin{aligned}
\tilde{\mathbf{v}}_{l}(\tilde{x}) & =\int_{\tilde{B}} \sum_{j=1}^{n} G_{l j}(\tilde{x}, \tilde{\xi}) \tilde{\mathbf{f}}_{j}(\tilde{\xi}) d \tilde{\xi}+\int_{\tilde{B}} G_{l, n+1}(\tilde{x}, \tilde{\xi}) \tilde{g}(\tilde{\xi}) d \tilde{\xi} \\
\tilde{q}(\tilde{x}) & =\int_{\tilde{B}} \sum_{j=1}^{n} G_{n+1, j}(\tilde{x}, \tilde{\xi}) \tilde{\mathbf{f}}_{j}(\tilde{\xi}) d \tilde{\xi}+\int_{\tilde{B}} G_{n+1, n+1}(\tilde{x}, \tilde{\xi}) \tilde{g}(\tilde{\xi}) d \tilde{\xi} .
\end{aligned}
$$

Moreover, there is a constant $C$ such that, for $\delta_{l, n+1}+|\alpha| \leq 1$ and $\delta_{n+1, j}+|\beta| \leq 1$,

$$
\left|D_{\tilde{x}}^{\alpha} D_{\tilde{\xi}}^{\beta} G_{l j}(\tilde{x}, \tilde{\xi})\right| \leq \begin{cases}C|\tilde{x}-\tilde{\xi}|^{-\kappa}, & \text { if } \kappa>0, \\ C \ln |\tilde{x}-\tilde{\xi}|, & \text { if } \kappa=0,\end{cases}
$$


where $\delta_{l, j}$ is Kronecker's delta and

$$
\kappa=n+\delta_{l, n+1}+|\alpha|+\delta_{n+1, j}+|\beta|-2 .
$$

In addition,

$$
G_{l j}(\tilde{x}, \tilde{\xi})=G_{j l}(\tilde{\xi}, \tilde{x}), \quad \tilde{x}, \tilde{\xi} \in \tilde{B}, 1 \leq l, j \leq n+1,
$$

and when $\phi$ is chosen as above,

$$
\int_{\tilde{B}} G_{n+1, j}(\tilde{x}, \tilde{\xi}) \phi(\tilde{x}) d \tilde{x}=0, \quad \tilde{\xi} \in \tilde{B}, 1 \leq j \leq n+1 .
$$

In the case of three space dimensions, these estimates can be found in Ros10a and MR10; cf. MR05. In the case $n=2$, Lemma 10 does not appear directly in the literature to our knowledge. In the case of Poisson's problem, a similar estimate for $n=2$ is found in Fro93 for generic convex domains. [NP94 contains Green's function estimates for elliptic scalar equations of order $2 m$ on (polygonal) cones in two space dimensions. The correct asymptotics for convex polygonal domains may be derived from these estimates (cf. Ros10b]), and the authors also state that "The passage to the case of elliptic systems entails only some notational complications." We shall thus also assume the results of Lemma 10 in the two-dimensional case.

Note that the constants $C$ in (4.2), (4.3), and (4.5) do not depend on the choice of the reference domain $\tilde{B}=\tilde{B}_{i}$, since $\tilde{B}_{i}$ lies in the finite set $\left\{\tilde{B}_{1}, \ldots, \tilde{B}_{M}\right\}$.

We finally remark that we only employ (4.5) with $\kappa=n$ and $\kappa=n-1$. The logarithmic estimate occurring when $\kappa=0$ is thus not used here; we only include it for the sake of completeness.

Remark 11. An alternative to using Green's function estimates is to employ $W_{q}^{1}$ type regularity estimates as $q \downarrow 1$. This was the approach taken in SL06] for proving gradient estimates for the Stokes system in the global maximum norm on convex polyhedral domains. The disadvantage of this approach is that it requires an unnatural restriction on the maximum interior dihedral angle when $n=3$. The a priori estimates of [GNS04, GNS05] suffer from the same restriction, which was subsequently overcome in GL10] by the use of sharp Green's function estimates. We similarly avoid this restriction by employing sharp Green's function estimates. Note also that the techniques we employ here could be easily used to extend the global $W_{\infty}^{1}$ a posteriori estimates of [SL06] to include convex polyhedral domains with no restriction on the maximum interior dihedral angle. In the context of Poisson's problem, we refer to Dem06 for a posteriori estimates and to GLRS09. for a priori estimates which similarly use sharp Green's function estimates to obtain pointwise gradient bounds on any convex polyhedral domain.

In two space dimensions, the use of $L_{q}$-type regularity estimates leads to optimal results with respect to domain geometry and thus provides a reasonable alternative to using Green's functions. The techniques of SL06 could be extended to prove local error estimates for $n=2$ as well.

4.2. Transformation to a reference domain. We now map $B$ to a reference domain $\tilde{B}$ by translating and scaling $\tilde{B}$ by a factor $c d$ for some $c_{2} \leq c \leq 1$ as in 2.2. Let $(\mathbf{v}, q)$ be the solution of (3.5). With slight abuse of notation, we assume $c=1$ and define $\tilde{\mathbf{v}}(\tilde{x})=d^{-1} \mathbf{v}(d \tilde{x}), \tilde{q}(\tilde{x})=q(d \tilde{x})$, and $\tilde{\delta}(\tilde{x})=\delta(d \tilde{x})$. Note that $\tilde{\delta}$ has radius of support $\rho / d$, since $\delta$ has radius of support $\rho$. We also have

$$
\mathcal{L}_{\tilde{B}}((\mathbf{w}, \lambda),(\tilde{\mathbf{v}}, \tilde{q}))=\left(\mathbf{w}, D_{\tilde{x}_{i}} \tilde{\delta} \mathbf{k}_{j}\right)+\left(\lambda, \tilde{\delta}-\tilde{\delta}_{B}\right) \quad \forall(\mathbf{w}, \lambda) \in V_{\tilde{B}} \times X_{\tilde{B}} ;
$$


recall that the maximum error is attained at $x_{0}$ in the $i$-th derivative of the $j$-th component of $\mathbf{e}_{\mathbf{u}}$.

Here we also make precise our definition of $\delta_{B}$ : we let $\tilde{\delta}_{B}(\tilde{x})=\phi(\tilde{x}) \int_{\tilde{B}} \tilde{\delta}(\tilde{y}) \mathrm{d} \tilde{y}$ and define $\delta_{B}$ correspondingly as $\delta_{B}(x)=\tilde{\delta}_{B}(x / d)$. Since $\int_{\tilde{B}} \phi(\tilde{x}) \mathrm{d} \tilde{x}=1$, we then have $\int_{\tilde{B}}\left(\tilde{\delta}-\tilde{\delta}_{B}\right) \mathrm{d} \tilde{x}=0$ as required. In addition, because $\phi$ is a fixed smooth function on $\tilde{B}$ and by a change of variables $\|\tilde{\delta}\|_{L_{1}(\tilde{B})} \leq C d^{-n}\|\delta\|_{L_{1}(B)} \leq C d^{-n}$, we have the estimate

$$
\left\|\delta_{B}\right\|_{L_{2}(B)}+d\left\|\nabla \delta_{B}\right\|_{L_{2}(B)} \leq C d^{n / 2}\left\|\tilde{\delta}_{B}\right\|_{H^{1}(\tilde{B})} \leq C d^{-n / 2},
$$

where $C$ depends only on $\tilde{B}$ (and thus ultimately only on $\Omega$ ).

4.3. Proof of Lemma 8, We can now prove regularity estimates for the localized adjoint problem (3.5).

Proof of (3.12). We begin by bounding $\|\mathbf{v}\|_{W_{1}^{1}(B)}+\|q\|_{L_{1}(B)}$. Letting $\tilde{x}_{0} \in \tilde{B}$ be the image of $x_{0} \in B$, we first compute

$$
\begin{aligned}
\|\mathbf{v}\|_{W_{1}^{1}(B)}+\|q\|_{L_{1}(B)} \leq & C d^{n}\left(\|\tilde{\mathbf{v}}\|_{W_{1}^{1}(\tilde{B})}+\|\tilde{q}\|_{L_{1}(\tilde{B})}\right) \\
\leq & C d^{n}\left(\|\tilde{\mathbf{v}}\|_{W_{1}^{1}\left(B_{2 \rho / d}\left(\tilde{x}_{0}\right)\right)}+\|\tilde{q}\|_{L_{1}\left(B_{2 \rho / d}\left(\tilde{x}_{0}\right)\right)}\right. \\
& \left.+\|\tilde{\mathbf{v}}\|_{W_{1}^{1}\left(\tilde{B} \backslash B_{2 \rho / d}\left(\tilde{x}_{0}\right)\right)}+\|\tilde{q}\|_{L_{1}\left(\tilde{B} \backslash B_{2 \rho / d}\left(\tilde{x}_{0}\right)\right)}\right) .
\end{aligned}
$$

Using (4.2) with $\tilde{\mathbf{f}}=D_{\tilde{x}_{i}} \tilde{\delta} \mathbf{k}_{j}, \tilde{g}=\tilde{\delta}-\tilde{\delta}_{B}$, (3.1), and (4.9), we next find that

$$
\begin{aligned}
& d^{n}\left(\|\tilde{\mathbf{v}}\|_{W_{1}^{1}\left(B_{2 \rho / d}\left(\tilde{x}_{0}\right)\right)}+\|\tilde{q}\|_{L_{1}\left(B_{2 \rho / d}\left(\tilde{x}_{0}\right)\right)}\right) \\
& \quad \leq C d^{n}\left(\frac{\rho}{d}\right)^{n / 2}\left(\|\tilde{\mathbf{v}}\|_{H^{1}\left(B_{2 \rho / d}\left(\tilde{x}_{0}\right)\right)}+\|\tilde{q}\|_{L_{2}\left(B_{2 \rho / d}\left(\tilde{x}_{0}\right)\right)}\right) \\
& \quad \leq C(\rho d)^{n / 2}\left(\|\tilde{\mathbf{v}}\|_{H_{0}^{1}(\tilde{B})}+\|\tilde{q}\|_{L_{2}(\tilde{B})}\right) \\
& \quad \leq C(\rho d)^{n / 2}\left(\|\tilde{\delta}\|_{L_{2}(\tilde{B})}+\left\|\tilde{\delta}-\tilde{\delta}_{B}\right\|_{L_{2}(\tilde{B})}\right) \\
& \quad \leq C \rho^{n / 2}\left(\|\delta\|_{L_{2}(B)}+\left\|\delta_{B}\right\|_{L_{2}(B)} \leq C .\right.
\end{aligned}
$$

Employing (4.4) and (4.5), and also using (4.7) while recalling that $\tilde{\delta}_{B}$ is a constant multiple of $\phi$, we have for $\tilde{x} \in \tilde{B} \backslash B_{2 \rho / d}\left(\tilde{x}_{0}\right)$, that

$$
\begin{aligned}
D_{\tilde{x}_{k}} \tilde{\mathbf{v}}_{l}(\tilde{x}) & =D_{\tilde{x}_{k}}\left(\int_{\tilde{B}} G_{l j}(\tilde{x}, \tilde{\xi}) D_{\tilde{\xi}_{i}} \tilde{\delta}(\tilde{\xi}) \mathrm{d} \tilde{\xi}+\int_{\tilde{B}} G_{l, n+1}(\tilde{x}, \tilde{\xi})\left(\tilde{\delta}-\tilde{\delta}_{B}\right)(\tilde{\xi}) \mathrm{d} \tilde{\xi}\right) \\
& =D_{\tilde{x}_{k}}\left(\int_{\tilde{B}} G_{l j}(\tilde{x}, \tilde{\xi}) D_{\tilde{\xi}_{i}} \tilde{\delta}(\tilde{\xi}) \mathrm{d} \tilde{\xi}+\int_{\tilde{B}} G_{l, n+1}(\tilde{x}, \tilde{\xi}) \tilde{\delta}(\tilde{\xi}) \mathrm{d} \tilde{\xi}\right) \\
& =\int_{B_{\rho / d}\left(\tilde{x}_{0}\right)} \tilde{\delta}(\tilde{\xi})\left(-D_{\tilde{x}_{k} \tilde{\xi}_{i}}^{2} G_{l j}(\tilde{x}, \tilde{\xi}) \mathrm{d} \tilde{\xi}+D_{\tilde{x}_{k}} G_{l, n+1}(\tilde{x}, \tilde{\xi})\right) \mathrm{d} \tilde{\xi} \\
& \leq\|\tilde{\delta}\|_{L_{1}(\tilde{B})}\left\|\left|D_{\tilde{x}_{k} \tilde{\xi}_{i}}^{2} G_{l j}(\tilde{x}, \cdot)\right|+\left|D_{\tilde{x}_{k}} G_{l, n+1}(\tilde{x}, \cdot)\right|\right\|_{L_{\infty}\left(B_{\rho / d}\left(\tilde{x}_{0}\right)\right)} \\
& \leq C d^{-n}\|\delta\|_{L_{1}(B)} \sup _{\tilde{\xi} \in B_{\rho / d}\left(\tilde{x}_{0}\right)}|\tilde{x}-\tilde{\xi}|^{-n} \leq C d^{-n}\left|\tilde{x}-\tilde{x}_{0}\right|^{-n} .
\end{aligned}
$$

For the term $D_{\tilde{x}_{k} \tilde{\xi}_{i}}^{2} G_{l j}(\tilde{x}, \cdot)$ we used that $1 \leq l, j \leq n,|\alpha|=|\beta|=1$, so that (4.5) applies with $\kappa=n$. For the term $D_{\tilde{x}_{k}} G_{l, n+1}(\tilde{x}, \cdot)$ we used that $|\alpha|=1$ and $|\beta|=0$, 
$l \leq n$, and $j=n+1$, so that again $\kappa=n$. We also used $\left|\tilde{x}-\tilde{x}_{0}\right| \leq|\tilde{x}-\tilde{\xi}|+\left|\tilde{\xi}-\tilde{x}_{0}\right| \leq$ $|\tilde{x}-\tilde{\xi}|+\rho / d \leq 2|\tilde{x}-\tilde{\xi}|$. Similarly,

$$
\tilde{q}(\tilde{x}) \leq C d^{-n}\left|\tilde{x}-\tilde{x}_{0}\right|^{-n}
$$

and

$$
\tilde{\mathbf{v}}(\tilde{x}) \leq C d^{-n}\left|\tilde{x}-\tilde{x}_{0}\right|^{1-n} .
$$

Thus, setting $r=\left|\tilde{x}-\tilde{x}_{0}\right|$ and transforming to polar coordinates leads to

$$
\begin{aligned}
& d^{n}\left(\|\tilde{\mathbf{v}}\|_{W_{1}^{1}\left(\tilde{B} \backslash B_{2 \rho / d}\left(\tilde{x}_{0}\right)\right)}+\|\tilde{q}\|_{L_{1}\left(\tilde{B} \backslash B_{2 \rho / d}\left(\tilde{x}_{0}\right)\right)}\right) \\
& \quad \leq C d^{n} d^{-n} \int_{2 \rho / d}^{\operatorname{diam}(\tilde{B})} r^{n-1} r^{-n} \mathrm{~d} r \leq C \ln \frac{d}{\rho} .
\end{aligned}
$$

Collecting (4.15) and (4.11) into (4.10) completes the proof of (3.12).

Proof of (3.13). We first complete the calculation for the term $\|\nabla \mathbf{v}\|_{W_{1}^{1}\left(B \backslash B_{d / 2}\right)}$, recalling that $B_{d / 2}=B_{c_{1} d / 2}\left(x_{0}\right) \cap B$; see (2.3). We set $c_{1}=1$ for simplicity. Transforming to $\tilde{B}$ and using Hölder's inequality, we have

$$
\|\nabla \mathbf{v}\|_{W_{1}^{1}\left(B \backslash B_{d / 2}\right)} \leq C d^{n}\|\nabla \tilde{\mathbf{v}}\|_{W_{1}^{1}\left(\tilde{B} \backslash B_{1 / 2}\left(\tilde{x}_{0}\right)\right)} \leq C d^{n}\|\nabla \tilde{\mathbf{v}}\|_{W_{\infty}^{1}\left(\tilde{B} \backslash B_{1 / 2}\left(\tilde{x}_{0}\right)\right)} .
$$

Note that since $\rho \leq c_{0} d$ for $c_{0}$ sufficiently small (here $c_{0}=\frac{1}{4}$ suffices), we have $\tilde{B} \backslash B_{1 / 2}\left(\tilde{x}_{0}\right) \cap B_{2 \rho / d}\left(\tilde{x}_{0}\right)=\emptyset$. Thus by (4.12), we have $|\nabla \tilde{\mathbf{v}}(\tilde{x})| \leq C d^{-n}$ for $\tilde{x} \in \tilde{B} \backslash B_{1 / 2}\left(\tilde{x}_{0}\right)$, which when inserted into the previous equation yields

$$
\|\nabla \mathbf{v}\|_{W_{1}^{1}\left(B \backslash B_{d / 2}\right)} \leq C,
$$

as desired. The bounds for $d^{-1}\|\mathbf{v}\|_{L_{1}\left(B \backslash B_{d / 2}\right)}$ and $\|q\|_{L_{1}\left(B \backslash B_{d / 2}\right)}$ similarly employ (4.13) and (4.14). Thus (3.13) is proved.

Proof of (3.14). Let $d_{0}=\frac{3}{2} \frac{\rho}{d}$ and $d_{j}=2^{j} \frac{\rho}{d}, j \geq 1$. Also, let $\Omega_{j}=\left\{\tilde{x} \in \tilde{B}: d_{j}<\mid \tilde{x}-\right.$ $\left.\tilde{x}_{0} \mid \leq d_{j+1}\right\}, j \geq 0$ and $\Omega_{j}^{\prime}=\Omega_{j-1} \cup \Omega_{j} \cup \Omega_{j+1}$; note that $\tilde{B} \backslash B_{2 \rho / d}\left(\tilde{x}_{0}\right)=\bigcup_{j=1}^{J} \Omega_{j}$ with $J \approx \ln \frac{d}{\rho}$. Finally, we let $\omega_{j}$ be a smooth cut-off function which is 1 on $\Omega_{j}, 0$ outside of $\Omega_{j-1} \cup \Omega_{j} \cup \Omega_{j+1}$, and which satisfies $\left\|D^{l} \omega_{j}\right\|_{L_{\infty}(\tilde{B})} \leq C d_{j}^{-l}, l=0,1,2$. Transforming to $\tilde{B}$, we then have

$$
\begin{aligned}
& \int_{B \backslash B_{2 \rho}\left(x_{0}\right)}\left|x-x_{0}\right|\left(|\nabla q(x)|+\left|D^{2} \mathbf{v}(x)\right|\right) \mathrm{d} x \\
& \leq C d^{n} \int_{\tilde{B} \backslash B_{2 \rho / d}\left(\tilde{x}_{0}\right)}\left|\tilde{x}-\tilde{x}_{0}\right|\left(|\nabla \tilde{q}(\tilde{x})|+\left|D^{2} \tilde{\mathbf{v}}(\tilde{x})\right|\right) \mathrm{d} \tilde{x} \\
& \leq C d^{n} \sum_{j=1}^{J} d_{j}^{n / 2+1}\left(\|\nabla \tilde{q}\|_{L_{2}\left(\Omega_{j}\right)}+\left\|D^{2} \tilde{\mathbf{v}}\right\|_{L_{2}\left(\Omega_{j}\right)}\right) \\
& \leq C d^{n} \sum_{j=1}^{J} d_{j}^{n / 2+1}\left(\left\|\nabla\left(\omega_{j} \tilde{q}\right)\right\|_{L_{2}(\tilde{B})}+\left\|D^{2}\left(\omega_{j} \tilde{\mathbf{v}}\right)\right\|_{L_{2}(\tilde{B})}\right) .
\end{aligned}
$$

We apply (4.3) with $\tilde{\mathbf{v}}$ replaced by $\omega_{j} \tilde{\mathbf{v}}$ and $\tilde{q}$ by $\omega_{j} \tilde{q}$. Then

$$
\tilde{\mathbf{f}}=-\Delta\left(\omega_{j} \tilde{\mathbf{v}}\right)-\nabla\left(\omega_{j} \tilde{q}\right)=-\tilde{\mathbf{v}} \Delta \omega_{j}-2 \nabla \tilde{\mathbf{v}} \nabla \omega_{j}-\tilde{q} \nabla \omega_{j},
$$

since $\omega_{j}(-\Delta \tilde{\mathbf{v}}-\nabla \tilde{q})=0$. Also,

$\tilde{g}=-\nabla \cdot\left(\omega_{j} \tilde{\mathbf{v}}\right)=-\omega_{j} \nabla \cdot \tilde{\mathbf{v}}-\tilde{\mathbf{v}} \cdot \nabla \omega_{j}=-\omega_{j}\left(\tilde{\delta}-\tilde{\delta}_{B}\right)-\tilde{\mathbf{v}} \cdot \nabla \omega_{j}=\omega_{j} \tilde{\delta}_{B}-\tilde{\mathbf{v}} \cdot \nabla \omega_{j}$, 
since $\operatorname{supp}(\tilde{\delta}) \subset B_{\rho / d}\left(\tilde{x}_{0}\right)$ and $\omega_{j}$ and $\tilde{\delta}$ thus have disjoint supports. Also, integration by parts yields $\int_{\tilde{B}} \tilde{g} \mathrm{~d} \tilde{x}=\int_{\tilde{B}} \omega_{j} \tilde{\mathbf{v}} \cdot \nabla 1 \mathrm{~d} \tilde{x}=0$, since $\tilde{\mathbf{v}}=0$ on $\partial \tilde{B}$. Employing (4.3), bounds for the derivatives of $\omega_{j}$, and Hölder's inequality then yields

$$
\begin{aligned}
C d^{n} \sum_{j=1}^{J} d_{j}^{n / 2+1}\left(\left\|\nabla\left(\omega_{j} \tilde{q}\right)\right\|_{L_{2}(\tilde{B})}+\left\|D^{2}\left(\omega_{j} \tilde{\mathbf{v}}\right)\right\|_{L_{2}(\tilde{B})}\right) \\
\leq C d^{n} \sum_{j=1}^{J} d_{j}^{n / 2+1}\left(\left\|\tilde{\mathbf{v}} \Delta \omega_{j}\right\|_{L_{2}(\tilde{B})}+2\left\|\nabla \tilde{\mathbf{v}} \nabla \omega_{j}\right\|_{L_{2}(\tilde{B})}\right. \\
\left.\quad+\left\|\tilde{q} \nabla \omega_{j}\right\|_{L_{2}(\tilde{B})}+\left\|\tilde{\mathbf{v}} \cdot \nabla \omega_{j}\right\|_{H^{1}(\tilde{B})}+\left\|\omega_{j} \tilde{\delta}_{B}\right\|_{H^{1}(\tilde{B})}\right) \\
\leq C d^{n} \sum_{j=1}^{J} d_{j}^{n}\left(d_{j}^{-1}\|\tilde{\mathbf{v}}\|_{L_{\infty}\left(\Omega_{j}^{\prime}\right)}+\|\nabla \tilde{\mathbf{v}}\|_{L_{\infty}\left(\Omega_{j}^{\prime}\right)}+\|\tilde{q}\|_{L_{\infty}\left(\Omega_{j}^{\prime}\right)}\right) \\
\quad+C d^{n} \sum_{j=1}^{J} d_{j}^{n / 2+1}\left(\left\|\nabla \tilde{\delta}_{B}\right\|_{L_{2}\left(\Omega_{j}^{\prime}\right)}+d_{j}^{-1}\left\|\tilde{\delta}_{B}\right\|_{L_{2}\left(\Omega_{j}^{\prime}\right)}\right) .
\end{aligned}
$$

Inequalities (4.12), (4.13), and (4.14) then yield

$$
\begin{gathered}
C d^{n} \sum_{j=1}^{J} d_{j}^{n}\left(d_{j}^{-1}\|\tilde{\mathbf{v}}\|_{L_{\infty}\left(\Omega_{j}^{\prime}\right)}+\|\nabla \tilde{\mathbf{v}}\|_{L_{\infty}\left(\Omega_{j}^{\prime}\right)}+\|\tilde{q}\|_{L_{\infty}\left(\Omega_{j}^{\prime}\right)}\right) \\
\leq C d^{n} \sum_{j=1}^{J} d^{-n}\left(d_{j}^{n-1} d_{j}^{1-n}+d_{j}^{n} d_{j}^{-n}\right) \leq C J \leq C \ln \frac{d}{\rho} .
\end{gathered}
$$

Employing (4.9) and the fact that $\sum_{j=1}^{J} d_{j}^{n / 2}$ is a geometric series with uniformly bounded largest term and is thus uniformly bounded, we also have

$$
d^{n} \sum_{j=1}^{J} d_{j}^{n / 2+1}\left(\left\|\nabla \tilde{\delta}_{B}\right\|_{L_{2}\left(\Omega_{j}^{\prime}\right)}+d_{j}^{-1}\left\|\tilde{\delta}_{B}\right\|_{L_{2}\left(\Omega_{j}^{\prime}\right)}\right) \leq C d^{n} \sum_{j=1}^{J} d_{j}^{n / 2} d^{-n} \leq C .
$$

Collecting the previous statements completes the proof of (3.14) and thus also of Lemma 8 .

\subsection{Regularity estimates for the Stokes system on general polyhedral}

domains. In order to bound the pollution error term $\left|\left(e_{p}, \omega \delta_{B}+\mathbf{v} \cdot \nabla \omega\right)\right|$ in Lemma 7 and thereby prove Lemma 9. we will carry out a second duality argument, but this time over the original domain $\Omega$. Our estimates employ $L_{p}$ regularity results for such dual problems, which we state now along with further similar estimates that we will use later in our development.

In the following two lemmas, we assume that $(\mathbf{z}, \lambda) \in V \times X$ solve

$$
\mathcal{L}((\mathbf{w}, q),(\mathbf{z}, \lambda))=(\mathbf{w}, \tilde{\mathbf{f}})+(q, \tilde{g}) \quad \forall(\mathbf{w}, q) \in V \times X,
$$

where we assume that $\int_{\Omega} \tilde{g}(x) \mathrm{d} x=0$. Other assumptions about $\tilde{\mathbf{f}}$ and $\tilde{g}$ will be made below. These lemmas are found in essentially the form below in SL06, Theorem 1.1, Theorem 1.3, and Remark 1.2 and summarize results from [BS95], KMR01, and MR07. 
Lemma 12. Let $\Omega \subset \mathbb{R}^{n}, n=2,3$, be a bounded Lipschitz domain. There exists $\epsilon>0$ such that if $\frac{3+\epsilon}{2+\epsilon}<q<3+\epsilon, \tilde{\mathbf{f}} \in W_{q}^{-1}(\Omega)^{n}$, and $\tilde{g} \in L_{q}(\Omega)$ with $\int_{\Omega} \tilde{g} d x=0$, then there is a unique solution $(\mathbf{z}, \lambda) \in W_{0}^{1, q}(\Omega)^{n} \times L_{q}(\Omega) / \mathbb{R}$ to (4.16). Moreover,

$$
\|\mathbf{z}\|_{W_{q}^{1}(\Omega)}+\|\lambda\|_{L_{q}(\Omega) / \mathbb{R}} \leq C\left(\|\tilde{\mathbf{f}}\|_{W_{q}^{-1}(\Omega)}+\|\tilde{g}\|_{L_{q}(\Omega)}\right) .
$$

Lemma 13. Let $\Omega \subset \mathbb{R}^{n}, n=2,3$, be a polyhedral domain and let $1<q \leq 4 / 3$. Suppose $\tilde{\mathbf{f}} \in L_{q}(\Omega)^{n}$ and $\tilde{g} \in W_{1}^{q}(\Omega)^{n}$ with $\int_{\Omega} \tilde{g} d x=0$. Then the unique weak solution $(\mathbf{z}, \lambda)$ to (4.16) satisfies

$$
\|\mathbf{z}\|_{W_{q}^{2}(\Omega)}+\|\lambda\|_{W_{q}^{1}(\Omega)} \leq C\left(\|\tilde{\mathbf{f}}\|_{L_{q}(\Omega)}+|\tilde{g}|_{W_{q}^{1}(\Omega)}\right) .
$$

Assume in addition that $n=2$ and that $\Omega$ is convex. Then (4.18) holds for some $q>2$ with $q$ sufficiently close to 2 , depending on the maximum interior angle of $\Omega$.

4.5. Proof of Lemma 9, We use a duality argument to bound the term $\mid\left(e_{p}, \omega \delta_{B}+\right.$ $\mathbf{v} \cdot \nabla \omega) \mid$ and thus prove Lemma 9 Let $\tilde{\mathbf{f}}=0, M=\frac{1}{|\Omega|} \int_{\Omega}\left(\omega \delta_{B}+\mathbf{v} \cdot \nabla \omega\right) \mathrm{d} x$, and $\tilde{g}=\omega \delta_{B}+\mathbf{v} \cdot \nabla \omega-M$. Noting that $\int_{\omega} e_{p} \mathrm{~d} x=0$, we have $\left(e_{p}, \omega \delta_{B}+\mathbf{v} \cdot \nabla \omega\right)=\left(e_{p}, \tilde{g}\right)$.

Let $(\mathbf{z}, \lambda) \in V \times X$ solve

$$
\mathcal{L}((\mathbf{w}, q),(\mathbf{z}, \lambda))=(q, \tilde{g}) \quad \forall(\mathbf{w}, q) \in V \times X .
$$

In strong form, we thus have $-\Delta \mathbf{z}-\nabla \lambda=0,-\nabla \cdot \mathbf{z}=\tilde{g}$. Then we have

$$
\left(e_{p}, \omega \delta_{B}+\mathbf{v} \cdot \nabla \omega\right)=\mathcal{L}\left(\left(\mathbf{e}_{\mathbf{u}}, e_{p}\right),(\mathbf{z}, \lambda)\right) .
$$

Standard manipulations for proving residual-type error estimates then yield

$$
\mathcal{L}\left(\left(\mathbf{e}_{\mathbf{u}}, e_{p}\right),(\mathbf{z}, \lambda)\right) \leq \mathbf{C}\left(\max _{\mathbf{T} \in \mathcal{T}_{\mathbf{h}}} \mathbf{h}_{\mathbf{T}} \eta_{\mathbf{1}, \infty(\mathbf{T})}\right)\left(|\mathbf{z}|_{\mathbf{W}_{1}^{2}(\boldsymbol{\Omega})}+|\lambda|_{\mathbf{W}_{1}^{1}(\boldsymbol{\Omega})}\right) .
$$

We now bound $|\mathbf{z}|_{W_{1}^{2}(\Omega)}+|\lambda|_{W_{1}^{1}(\Omega)}$. Recalling from $\$ 2.2$ that $\omega \in C_{0}^{\infty}\left(B_{c_{1} d}\left(x_{0}\right)\right)$, we let $B_{K d}=B_{K c_{1} d}\left(x_{0}\right)$ for $K>0$ and note that

$$
\begin{aligned}
& |\mathbf{z}|_{W_{1}^{2}(\Omega)}+|\lambda|_{W_{1}^{1}(\Omega)} \\
& \quad \leq|\mathbf{z}|_{W_{1}^{2}\left(B_{4 d}\right)}+|\lambda|_{W_{1}^{1}\left(B_{4 d}\right)}+|\mathbf{z}|_{W_{1}^{2}\left(\Omega \backslash B_{4 d}\right)}+|\lambda|_{W_{1}^{1}\left(\Omega \backslash B_{4 d}\right)} .
\end{aligned}
$$

Fixing $q_{0}$ with $\frac{4}{3} \geq q_{0}>1$, recalling that $\left|D^{j} \omega\right| \leq C d^{-j}$, recalling that $\omega \equiv 1$ on $B_{d / 2}$, and finally applying Hölder's inequality, we compute that

$$
\begin{aligned}
&|\mathbf{z}|_{W_{1}^{2}\left(B_{4 d}\right)}+|\lambda|_{W_{1}^{1}\left(B_{4 d}\right)} \leq C d^{n\left(1-1 / q_{0}\right)}\left(|\mathbf{z}|_{W_{q_{0}}^{2}\left(B_{4 d}\right)}+|\lambda|_{W_{q_{0}}^{1}\left(B_{4 d}\right)}\right) \\
& \leq C d^{n\left(1-1 / q_{0}\right)}\|\nabla \tilde{g}\|_{L_{q_{0}}(\Omega)} \\
& \leq C d^{n\left(1-1 / q_{0}\right)}\left\|\nabla\left(\omega \delta_{B}+\mathbf{v} \cdot \nabla \omega\right)\right\|_{L_{q_{0}}(\Omega)} \\
& \leq C\left(d^{n-2}\|\mathbf{v}\|_{L_{\infty}\left(B_{d} \backslash B_{d / 2}\right)}+d^{n-1}\|\nabla \mathbf{v}\|_{L_{\infty}\left(B_{d} \backslash B_{d / 2}\right)}\right. \\
&\left.+d^{n / 2}\left\|\nabla\left(\omega \delta_{B}\right)\right\|_{L_{2}(B)}\right) \\
& \leq C\left(d^{n-2}\|\mathbf{v}\|_{L_{\infty}\left(B_{d} \backslash B_{d / 2}\right)}+d^{n-1}\|\nabla \mathbf{v}\|_{L_{\infty}\left(B_{d} \backslash B_{d / 2}\right)}\right. \\
&\left.+d^{n / 2-1}\left\|\delta_{B}\right\|_{L_{2}(B)}+d^{n / 2}\left\|\nabla \delta_{B}\right\|_{L_{2}(B)}\right) .
\end{aligned}
$$


Next, we let $d_{j}=\left(4 c_{1} d\right) 2^{j-1}, j \geq 0$, and $\Omega_{j}=\left\{x \in \Omega: d_{j} \leq\left|x-x_{0}\right| \leq d_{j+1}\right\}$, $j \geq 0$. We then let $\Omega_{j}^{\prime}=\left\{x \in \Omega: d_{j-1} \leq\left|x-x_{0}\right| \leq d_{j+2}\right.$, and let $\omega_{j}$ be a smooth cut-off function which is 1 on $\Omega_{j}, 0$ outside of $\Omega_{j}^{\prime}$, and which satisfies $\left\|D^{k} \omega_{j}\right\| \leq$ $C d_{j}^{-k}, k \geq 0$. Recall that $-\Delta \mathbf{z}-\nabla \lambda=0$ and note that the support of $\omega_{j}$ does not overlap with the support of $\omega \delta_{B}+\mathbf{v} \cdot \nabla \omega$ for $j \geq 0$. Thus $-\Delta\left(\omega_{j} \mathbf{z}\right)-\nabla\left(\omega_{j} \lambda\right)=$ $-2 \nabla \omega_{j} \nabla \mathbf{z}-\mathbf{z} \Delta \omega_{j}-\lambda \nabla \omega_{j}$, and $-\nabla \cdot\left(\omega_{j} \mathbf{z}\right)=-\omega_{j} \nabla \cdot \mathbf{z}-\mathbf{z} \nabla \omega_{j}=-\omega_{j} M-\mathbf{z} \nabla \omega_{j}$.

Choosing $J$ to be the smallest integer so that $d_{j} \geq \operatorname{diam}(\Omega)$ (so that $J \approx$ $\left.\log _{2} \frac{\operatorname{diam}(\Omega)}{d}\right)$, we apply Hölder's inequality and (4.18) with $q_{0}$ as above to compute that for $q_{2} \geq q_{1} \geq q_{0}$

$$
\begin{aligned}
|\mathbf{z}|_{W_{1}^{2}\left(\Omega \backslash B_{4 d}\right)}+|\lambda|_{W_{1}^{1}\left(\Omega \backslash B_{4 d}\right)} \leq \sum_{j=1}^{J}\left(\left|\omega_{j} \mathbf{z}\right|_{W_{1}^{2}\left(\Omega_{j}^{\prime}\right)}+\left|\omega_{j} \lambda\right|_{W_{1}^{1}\left(\Omega_{j}^{\prime}\right)}\right) \\
\leq \sum_{j=1}^{J} d_{j}^{n\left(1-1 / q_{0}\right)}\left(\left\|2 \nabla \omega_{j} \nabla \mathbf{z}+\mathbf{z} \Delta \omega_{j}+\lambda \nabla \omega_{j}\right\|_{L_{q_{0}}(\Omega)}\right. \\
\left.\quad+\left\|\nabla\left(\omega_{j} M+\mathbf{z} \nabla \omega_{j}\right)\right\|_{L_{q_{0}}(\Omega)}\right) \\
\leq \sum_{j=1}^{J} d_{j}^{n\left(1-1 / q_{0}\right)}\left(d_{j}^{-2}\|\mathbf{z}\|_{L_{q_{0}}\left(\Omega_{j}^{\prime}\right)}+d_{j}^{-1}\|\nabla \mathbf{z}\|_{L_{q_{0}}\left(\Omega_{j}^{\prime}\right)}+d_{j}^{-1}\|\lambda\|_{L_{q_{0}}\left(\Omega_{j}^{\prime}\right)}\right) \\
\quad+\sum_{j=1}^{J} d_{j}^{n-1}|M| \\
\leq\left(\|\nabla \mathbf{z}\|_{L_{q_{1}}(\Omega)}+\|\lambda\|_{L_{q_{1}}(\Omega)}\right) \sum_{j=1}^{J} d_{j}^{-1+n\left(1-1 / q_{1}\right)} \\
\quad+\|\mathbf{z}\|_{L_{q_{2}}(\Omega)} \sum_{j=1}^{J} d_{j}^{-2+n\left(1-1 / q_{2}\right)}+|M| .
\end{aligned}
$$

In the next-to-last inequality we have used the fact that $M$ is a constant to compute $d_{j}^{n\left(1-1 / q_{0}\right)}\left\|\nabla\left(\omega_{j} M\right)\right\|_{L_{q_{0}}(\Omega)} \leq C d_{j}^{n-1}$ and in the last inequality we have noted that $\sum_{j=1}^{J} d_{j}^{n-1}$ is a uniformly bounded geometric sum.

We next choose $q_{1}=\frac{3-\mu}{2}$, where $\mu$ is fixed and sufficiently small so that $q_{1}>\frac{3+\epsilon}{2+\epsilon}$ with $\epsilon$ as in Lemma 12. Noting that $q_{1}<n$ for $n=2,3$, we choose $q_{2}=\frac{n q_{1}}{n-q_{1}}$. Noting that $-2+\left(1-1 / q_{2}\right)=-1+\left(1-1 / q_{1}\right)$ and using the Sobolev inquality $\|\mathbf{z}\|_{L_{q_{2}}(\Omega)} \leq C\|\nabla \mathbf{z}\|_{L_{q_{1}}(\Omega)}$, we have

$$
\|\mathbf{z}\|_{L_{q_{2}}(\Omega)} \sum_{j=1}^{J} d_{j}^{-2+n\left(1-1 / q_{2}\right)} \leq C\|\nabla \mathbf{z}\|_{L_{q_{1}}(\Omega)} \sum_{j=1}^{J} d_{j}^{-1+n\left(1-1 / q_{1}\right)} .
$$

Note in addition that $|M| \leq\left\|\omega \delta_{B}+\mathbf{v} \cdot \nabla \omega\right\|_{L_{q_{1}}(\Omega)}$. Inserting this inequality and (4.25) into (4.24) while employing the regularity result (4.17) and finally employing 
Hölder's inequality yields

$$
\begin{aligned}
& |\mathbf{z}|_{W_{1}^{2}\left(\Omega \backslash B_{4 d}\right)}+|\lambda|_{W_{1}^{1}\left(\Omega \backslash B_{4 d}\right)} \\
& \leq\left(\|\nabla \mathbf{z}\|_{L_{q_{1}}(\Omega)}+\|\lambda\|_{L_{q_{1}}(\Omega)}\right) \sum_{j=1}^{J} d_{j}^{-1+n\left(1-1 / q_{1}\right)}+|M| \\
& \leq C\|\tilde{g}\|_{L_{q_{1}}(\Omega)} \sum_{j=1}^{J} d_{j}^{-1+n\left(1-1 / q_{1}\right)}+|M| \\
& \leq C\left(\left\|\omega \delta_{B}+\mathbf{v} \cdot \nabla \omega\right\|_{L_{q_{1}}(\Omega)}\right) \sum_{j=1}^{J} d_{j}^{-1+n\left(1-1 / q_{1}\right)} \\
& \leq C\left(d^{-1}\|\mathbf{v}\|_{L_{q_{1}}\left(B \backslash B_{d / 2}\right)}+\left\|\omega \delta_{B}\right\|_{L_{q_{1}}(B)}\right) \sum_{j=1}^{J} d_{j}^{-1+n\left(1-1 / q_{1}\right)} \\
& \leq C\left(d^{-1+n / q_{1}}\|\mathbf{v}\|_{L_{\infty}\left(B \backslash B_{d / 2}\right)}+d^{n / q_{1}-n / 2}\left\|\omega \delta_{B}\right\|_{L_{2}(B)}\right) \sum_{j=1}^{J} d_{j}^{-1+n\left(1-1 / q_{1}\right)} .
\end{aligned}
$$

Collecting (4.26) and (4.23) into (4.22) then yields that for $q_{1}=\frac{3-\mu}{2}$,

$$
\begin{aligned}
& |\mathbf{z}|_{W_{1}^{2}(\Omega)}+|\lambda|_{W_{1}^{1}(\Omega)} \\
& \leq C\left(d^{n-2}+d^{-1+n / q_{1}} \sum_{j=1}^{J} d_{j}^{-1+n\left(1-1 / q_{1}\right)}\right)\|\mathbf{v}\|_{L_{\infty}\left(B \backslash B_{d / 2}\right)} \\
& \quad+C\left(d^{n / q_{1}-n / 2} \sum_{j=1}^{J} d_{j}^{-1+n\left(1-1 / q_{1}\right)}+d^{n / 2-1}\right)\left\|\delta_{B}\right\|_{L_{2}(B)} \\
& \quad+C d^{n-1}\|\nabla \mathbf{v}\|_{L_{\infty}\left(B_{d} \backslash B_{d / 2}\right)}+C d^{n / 2}\left\|\nabla \delta_{B}\right\|_{L_{2}\left(B_{d}\right)} .
\end{aligned}
$$

We easily compute from the change of variables $\mathbf{v}(d \tilde{x})=d \tilde{\mathbf{v}}$ and from (4.12), (4.14) that $d^{-1}\|\mathbf{v}\|_{L_{\infty}\left(B \backslash B_{d / 2}\right)}+\|\nabla \mathbf{v}\|_{L_{\infty}\left(B_{d} \backslash B_{d / 2}\right)} \leq C d^{-n}$. Inserting this inequality along with (4.9) into (4.27) yields

$$
\begin{aligned}
& |\mathbf{z}|_{W_{1}^{2}(\Omega)}+|\lambda|_{W_{1}^{1}(\Omega)} \leq C\left(d^{-1}+d^{n / q_{1}-n} \sum_{j=1}^{J} d_{j}^{-1+n\left(1-1 / q_{1}\right)}\right) \\
& =C d^{-1}\left(1+d^{1-n+n / q_{1}} \sum_{j=1}^{J} d_{j}^{-1+n\left(1-1 / q_{1}\right)}\right) \\
& =C d^{-1}\left(1+\sum_{j=1}^{J}\left(\frac{d}{d_{j}}\right)^{1-n+n / q_{1}}\right)
\end{aligned}
$$

We now recall that $q_{1}=\frac{3-\mu}{2}$, so that for $n=2,3$ we have $1-n+n / q_{1}>0$. Since $\frac{d}{d_{j}} \sim 2^{-j}$, the above sum is thus bounded by a constant, and we finally have

$$
|\mathbf{z}|_{W_{1}^{2}(\Omega)}+|\lambda|_{W_{1}^{1}(\Omega)} \leq C d^{-1}
$$




\section{Proof of Corollary 2; Regularity of $(\mathbf{u}, p)$ and Removal OF THE REGULARIZATION PENALTY}

In this section we state precise local regularity results for the solution $(\mathbf{u}, p)$ on $D$ and use them to reabsorb the "regularization penalty" $|\mathbf{u}|_{C^{1, \beta}\left(\overline{B_{\rho}}\right)}+|p|_{C^{0, \beta}\left(\overline{B_{\rho}}\right)}$.

5.1. Global regularity on convex polyhedral domains. Before proving local regularity estimates, we first state global regularity estimates on convex polyhedral domains. In space dimension $n=2$, we are able to use $W_{q}^{2} \times W_{q}^{1}$ regularity for $q>2$ along with a Sobolev imbedding in order to obtain $C^{1, \beta} \times C^{0, \beta}$ regularity. This approach fails in three space dimensions, since $W_{q}^{2}$ imbeds into $C^{1, \beta}$ only if $q>3$ but obtaining $W_{q}^{2} \times W_{q}^{2}$ regularity for $q>3$ on convex polyhedral domains requires that all edge opening angles be less than $\frac{3 \pi}{4}$. Instead we state Schauder estimates directly. These estimates hold for any convex polyhedral domain, but require that the compressibility $g$ be 0 on a neighborhood of all edges of $\partial \Omega$. We are unaware of similar two-dimensional Schauder estimates in the literature, thus the necessity of using different regularity results depending on space dimension.

Lemma 14. Assume that $\tilde{B}$ is a convex polygonal or polyhedral domain in $\mathbb{R}^{n}$, $n=2,3 . \quad$ Let $(\tilde{\mathbf{u}}, \tilde{p}) \in V_{\tilde{B}} \times X_{\tilde{B}}$ solve $\mathcal{L}_{\tilde{B}}((\tilde{\mathbf{u}}, \tilde{p}),(\tilde{\mathbf{v}}, \tilde{\lambda}))=(\tilde{\mathbf{f}}, \tilde{\mathbf{v}})+(\tilde{\lambda}, \tilde{g})$ for all $(\tilde{\mathbf{v}}, \tilde{\lambda}) \in V_{\tilde{B}} \times X_{\tilde{B}}$. Assume that $\int_{\tilde{B}} \tilde{g} d x=0$ and, in addition, that $\tilde{g}=0$ on a neighborhood of all edges of $\partial \tilde{B}$ when $n=3$. Then for $q>n$, sufficiently close to $n$ (depending on the maximum edge opening angle of $\tilde{B}$ ), and $\beta=1-\frac{n}{q}$,

$$
\|\tilde{\mathbf{u}}\|_{C^{1, \beta}(\overline{\tilde{B}})}+\|\tilde{p}\|_{C^{0, \beta}(\overline{\tilde{B}})} \leq C\left(\|\tilde{\mathbf{f}}\|_{L_{q}(\tilde{B})}+\|\tilde{g}\|_{W_{q}^{1}(\tilde{B})}\right) .
$$

Proof. First we let $n=2$. Using the Sobolev imbeddings $W_{q}^{m+1}(\Omega) \hookrightarrow C^{m, \beta}(\overline{\tilde{B}})$ $\left(m=0,1, \beta=1-\frac{n}{q}\right.$; cf. Lemma 5.17 of Ada75) and then a $W_{q}^{2} \times W_{q}^{1}$ regularity estimate with $q>2$ but sufficiently close to 2 as in Lemma 13, we obtain

$$
\begin{aligned}
\|\tilde{\mathbf{u}}\|_{C^{1, \beta}(\tilde{B})}+\|\tilde{p}\|_{C^{0, \beta}(\tilde{B})} & \leq C\left(\|\tilde{\mathbf{u}}\|_{W_{q}^{2}(\tilde{B})}+\|\tilde{p}\|_{W_{q}^{1}(\tilde{B})}\right) \\
& \leq C\left(\|\tilde{\mathbf{f}}\|_{L_{q}(\tilde{B})}+\|\tilde{g}\|_{W_{q}^{1}(\tilde{B})}\right) .
\end{aligned}
$$

In the case $n=3$, it is stated in [MR06], $\S 6.4$, that if $(\tilde{\mathbf{f}}, \tilde{g}) \in\left[C^{-1, \beta}(\overline{\tilde{B}})\right]^{3} \times$ $C^{0, \beta}(\overline{\tilde{B}})$ with $\beta>0$ sufficiently small, then $(\tilde{\mathbf{u}}, \tilde{p}) \in\left[C^{1, \beta}(\overline{\tilde{B}})\right]^{3} \times C^{0, \beta}(\overline{\tilde{B}})$. When combined with Fredholm properties of the associated operators (cf. [MR10]), this statement implies the regularity estimate

$$
\|\tilde{\mathbf{u}}\|_{\left[C^{1, \beta}(\overline{\tilde{B}})\right]^{3}}+\|\tilde{p}\|_{C^{0, \beta}(\overline{\tilde{B}})} \leq C\left(\|\tilde{\mathbf{f}}\|_{\left[C^{-1, \beta}(\overline{\tilde{B}})\right]^{3}}+\|\tilde{g}\|_{C^{0, \beta}(\tilde{\tilde{B}})}\right) .
$$

Here $\tilde{\mathbf{f}} \in\left[C^{-1, \beta}(\overline{\tilde{B}})\right]^{3}$ if $\tilde{\mathbf{f}}=\mathbf{f}^{(0)}+\sum_{j=1}^{3} \partial_{x_{j}} \mathbf{f}^{(j)}$, where $\mathbf{f}^{(j)} \in\left[C^{0, \beta}(\overline{\tilde{B}})\right]^{3}, j=0, \ldots, 3$. That is, $\tilde{\mathbf{f}}$ is a linear combination of distributional derivatives of $C^{0, \beta}$ functions; cf. equation (71) of MR06. The corresponding $C^{-1, \beta}$ norm is given by the infimum over all such representations of $\tilde{\mathbf{f}}$ of the quantity $\sum_{j=0}^{3}\left\|\mathbf{f}^{(j)}\right\|_{C^{0, \beta}(\bar{B})}$ (cf. Ama00, $\S 2$ ). We choose $\beta$ so that (5.3) holds and then let $q=\frac{n}{1-\beta}$.

The last step in the proof is to use the natural Sobolev imbedding $L_{q}(\tilde{B}) \hookrightarrow$ $C^{-1, \beta}(\overline{\tilde{B}})$, which completes the proof when combined with $(\underline{5.3})$. Negative-order Sobolev spaces are not entirely common in the literature, however, and we are unaware of a direct reference to this imbedding and so establish its validity as 
follows. Note first that for $\beta \notin \mathbb{N}, C^{-1, \beta}\left(\mathbb{R}^{n}\right)$ is equivalent to the Besov space $B_{\infty, \infty}^{-1+\beta}\left(\mathbb{R}^{n}\right)$ (cf. (3.5) of Ama00), which in turn satisfies the embedding $L_{q}\left(\mathbb{R}^{n}\right) \hookrightarrow$ $B_{\infty, \infty}^{-1+\beta}\left(\mathbb{R}^{n}\right)$ (cf. RS96, Theorem on p. 31, equation (2)). Extending $\tilde{\mathbf{f}}$ boundedly to $\mathbb{R}^{3}$ in $\left[L_{q}\right]^{3}$ and employing these relationships in turn completes the proof.

5.2. Local regularity. We prove a regularity bound for $|\mathbf{u}|_{C^{1, \beta}\left(\overline{B_{\rho}}\right)}+|p|_{C^{0, \beta}\left(\overline{B_{\rho}}\right)}$. As previously let $B \subset \Omega$ be the local scaled reference domain containing $x_{0}$, let $\widetilde{B}$ be the corresponding fixed unit reference domain, and let $\omega$ be the cut-off function about $x_{0}$. Denote by $\widetilde{\omega \mathbf{u}}$ and $\widetilde{\omega p}$ the natural transformations of $\omega \mathbf{u}$ and $\omega p$ to $\widetilde{B}$, that is, $\widetilde{\omega \mathbf{u}}(\widetilde{x})=d^{-1} \mathbf{u}(d \widetilde{x}) \omega(d \widetilde{x})$ and $\widetilde{\omega p}(\widetilde{x})=\omega(d \widetilde{x}) p(d \widetilde{x})$. Also, $\tilde{\mathbf{u}}(\tilde{x})=d^{-1} \mathbf{u}(d \tilde{x})$ and $\tilde{p}(\tilde{x})=p(d \tilde{x})$. Then $-\Delta \tilde{\mathbf{u}}(\tilde{x})+\nabla \tilde{p}(\tilde{x})=d \mathbf{f}(d \tilde{x})$, and $\nabla \cdot \tilde{\mathbf{u}}(\tilde{x})=g(d \tilde{x})$ in $\tilde{B}$. Also, the assumption that $\omega \equiv 1$ on $B_{c_{1} d / 2}\left(x_{0}\right)$ and $\rho \leq c_{0} d$ for $c_{0}$ sufficiently small implies that $\omega \equiv 1$ on $B_{\rho}$. Employing Lemma 14 with $q$ and $\beta=1-\frac{q}{n}$ chosen as above and performing elementary manipulations yields

$$
\begin{aligned}
&|\mathbf{u}|_{C^{1, \beta}\left(\overline{B_{\rho}}\right)}+|p|_{C^{0, \beta}\left(\overline{B_{\rho}}\right)} \\
& \leq C d^{-\beta}\left(|\widetilde{\mathbf{u} \omega}|_{C^{1, \beta}(\overline{\widetilde{B}})}+|\widetilde{\omega p}|_{C^{0, \beta}(\overline{\widetilde{B}})}\right) \\
& \leq C d^{-\beta}\left(\|-\Delta(\widetilde{\omega \mathbf{u}})+\nabla(\widetilde{\omega p})\|_{L_{q}(\tilde{B})}+\|\nabla \cdot(\widetilde{\omega \mathbf{u}})\|_{W_{q}^{1}(\tilde{B})}\right) \\
& \leq C d^{-\beta-n / q}\left(d\|\mathbf{f}\|_{L_{q}(B)}+\|\nabla \mathbf{u}\|_{L_{q}(B)}+d^{-1}\|\mathbf{u}\|_{L_{q}(B)}+\|p\|_{L_{q}(B)}\right. \\
&\left.+\|g\|_{L_{q}(B)}+d\|\nabla g\|_{L_{q}(B)}\right) \\
&= C d^{-1}\left(d\|\mathbf{f}\|_{L_{q}(B)}+\|\nabla \mathbf{u}\|_{L_{q}(B)}+d^{-1}\|\mathbf{u}\|_{L_{q}(B)}+\|p\|_{L_{q}(B)}\right. \\
&\left.+\|g\|_{L_{q}(B)}+d\|\nabla g\|_{L_{q}(B)}\right) \\
&= C\left(\|\mathbf{f}\|_{L_{q}(B)}+\|\nabla g\|_{L_{q}(B)}+d^{-1}\|g\|_{L_{q}(B)}+d^{-1}\|\nabla \mathbf{u}\|_{L_{q}(B)}\right. \\
&\left.+d^{-1}\|p\|_{L_{q}(B)}+d^{-2}\|\mathbf{u}\|_{L_{q}(B)}\right) .
\end{aligned}
$$

Assuming, without loss of generality, that $q>n$ is chosen so that the $W_{q}^{1} \times L_{q}$ regularity estimate of Lemma 12 applies, we finally employ the regularity estimate (4.17) on the whole domain $\Omega$ in order to obtain the following lemma.

Lemma 15. Let $B_{\rho}$ be as in Theorem 1 and $B$ as above and let $\mathbf{f}$ and $g$ satisfy the assumptions in Corollary 2. Then for $q>n$, sufficiently close to $n$, and $\beta=1-\frac{n}{q}$, the solution of (1.1) satisfies

$$
\begin{aligned}
|\mathbf{u}|_{C^{1, \beta}\left(\overline{B_{\rho}}\right)} & +|p|_{C^{0, \beta}\left(\overline{B_{\rho}}\right)} \\
& \leq C\left(\|\mathbf{f}\|_{L_{q}(B)}+\|g\|_{W_{q}^{1}(B)}+d^{-2}\|\mathbf{f}\|_{W_{q}^{-1}(\Omega)}+d^{-2}\|g\|_{L_{q}(\Omega)}\right) .
\end{aligned}
$$

Remark 16. It is possible to sharpen (5.5) slightly by following Corollary 1.4 of SL06]. In particular, it is possible to bound $d^{-2}\|\mathbf{u}\|_{L_{q}(\Omega)}$ by $d^{-2}\left(\|\mathbf{f}\|_{W_{\tilde{q}}^{-2}(\Omega)}+\right.$ $\left.\|g\|_{\left(W_{\tilde{p}}^{1}(\Omega) / \mathbb{R}\right)^{\prime}}\right)$ for some $\tilde{q}$ sufficiently large, where $\frac{1}{\tilde{q}}+\frac{1}{\tilde{p}}=1$ and $\left(W_{\tilde{p}}^{1}(\Omega) / \mathbb{R}\right)^{\prime}$ is the dual space to $W_{\tilde{q}}^{1}(\Omega) / \mathbb{R}$. Using such an argument allows us multiply factors of $\|\mathbf{f}\|_{W_{q}^{-1}(\Omega)}+\|g\|_{L_{q}(\Omega)}$ by $d^{-1}$ instead of $d^{-2}$ in our final result. This makes little difference in the end, since dependence on $d$ is only logarithmic, so we do not pursue this line of argument. 
5.3. Proof of Corollary 2, Let $F(\mathbf{f}, g, d)$ and $\mathcal{E}$ be as in Corollary 2, Assume first that $\mathcal{E}=0$. Then we may let $\rho \rightarrow 0$ in Theorem 1 , since the factor $\ln \frac{d}{\rho}$ is not present. Combining the result with Lemma 3 yields (1.7).

If $\mathcal{E} \neq 0$, then we let $\rho=\tilde{\rho}=\min \left(c_{0} d, c_{0} \underline{h},(\mathcal{E} / F(\mathbf{f}, g, d))^{1 / \beta}\right)$ in Theorem 1 , Hence $\rho^{\beta} \leq \mathcal{E} / F(\mathbf{f}, g, d)$. Employing this inequality along with (5.5) yields

$$
\rho^{\beta}\left(|\mathbf{u}|_{C^{1, \beta}\left(\overline{D_{\rho}}\right)}+|p|_{C^{0, \beta}\left(\overline{D_{\rho}}\right)}\right) \leq C \mathcal{E} .
$$

Inserting (5.6) into (1.6) along with (2.5) completes the proof of (1.8), and thus of Corollary 2 ,

\section{Computational example}

6.1. Algorithm. In our tests we employ the standard adaptive finite element algorithm given by

$$
\text { solve } \rightarrow \text { estimate } \rightarrow \text { mark } \rightarrow \text { refine. }
$$

Given $D, d$, and $D_{d}$ as above, we let

$$
\eta(T)= \begin{cases}\frac{h_{T}}{h_{T}+\operatorname{dist}(T, D)} \eta_{1, \infty}(T), & T \cap D_{d} \neq \emptyset, \\ \frac{h_{T}}{d} \eta_{1, \infty}(T), & T \cap D_{d}=\emptyset .\end{cases}
$$

We employ a maximum strategy in the "mark" step of the algorithm. More precisely, we mark an element $T \in \mathcal{T}_{h}$ for refinement if

$$
\eta(T) \geq 0.5 \max _{T^{\prime} \in \mathcal{T}_{h}} \eta\left(T^{\prime}\right) .
$$

Note that greater efficiency can at times be obtained by calibrating constants more carefully in (6.1), i.e., by weighting residual contributions $\frac{h_{T}}{d} \eta_{1, \infty}(T)$ from elements $T \in \mathcal{T}_{h} \backslash \mathcal{T}_{D_{d}}$ by a different constant than residual contributions from elements in $\mathcal{T}_{D_{d}}$. Fine-tuning the algorithm in this fashion does not affect rates of convergence and is explored more thoroughly in Dem07, so we do not consider it further here.

We use the polynomial degree $k=2$ in our tests. The computations were carried out using the finite element toolbox ALBERTA (cf. SS05]).

\subsection{Test function and subdomain. Let}

$$
w(x)= \begin{cases}1+384(x-1)(x-0.5)^{5}-64(x-0.5)^{6}, & x \geq 0.5, \\ 1, & 0 \leq x<0.5, \\ w(-x), & x<0 .\end{cases}
$$

Note that $w \in W_{\infty}^{3}(\mathbb{R})$. Letting $(r, \phi)$ be polar coordinates, we also define

$$
\gamma(r, \theta)=r^{1.5}(3 \sin (\theta / 2)-\sin (3 \theta / 2)) .
$$

Finally, we let

$$
\begin{aligned}
& u_{1}(x, y)=\frac{\partial}{\partial y}(w(x) w(y) \gamma(r(x, y), \theta(x, y))), \\
& u_{2}(x, y)=-\frac{\partial}{\partial x}(w(x) w(y) \gamma(r(x, y), \theta(x, y))),
\end{aligned}
$$

and

$$
\mathbf{u}(x, y)=\left(u_{1}(x, y), u_{2}(x, y)\right)
$$


Then $\mathbf{u}$ satisfies homogenous Dirichlet boundary conditions on $\partial \Omega$ and also satisfies $\nabla \cdot \mathbf{u}=0$ in $\Omega$. Finally, we let

$$
p(r, \theta)=-6 r^{-0.5} \cos (\theta / 2) .
$$

Then $-\Delta \mathbf{u}+\nabla p=0$ for $r<0.5$. For $r>0.5$, we set $\mathbf{f}=-\Delta \mathbf{u}+\nabla p$. Note that the most singular part of $\mathbf{u}$ behaves like $r^{0.5}$, so $\mathbf{u} \in H^{3 / 2-\epsilon}(\Omega)$ for any $\epsilon>0$. Also, $\nabla \mathbf{u}$ is not bounded near the origin, but $\mathbf{u}$ is in fact smooth (infinitely differentiable) on any open subset of $\Omega$ not abutting the origin.

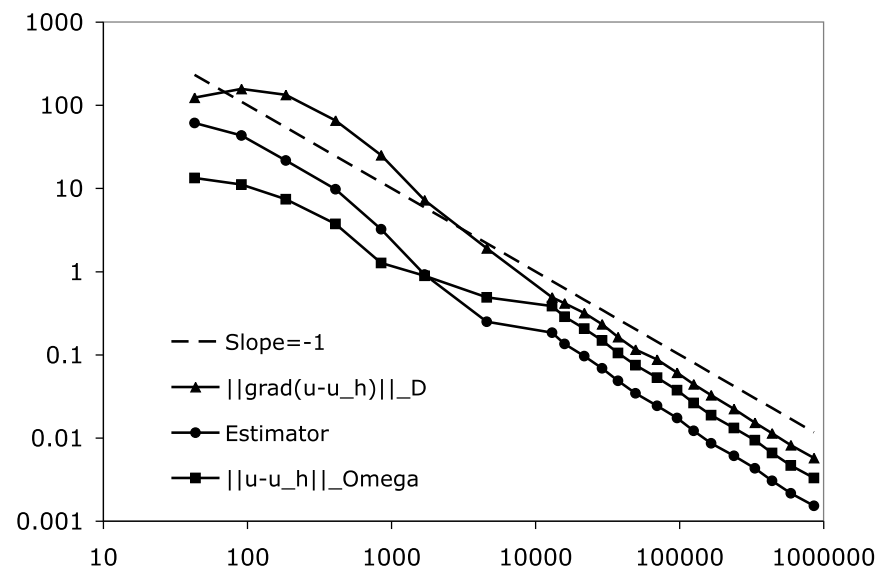

Figure 4. Target error $\left\|\nabla\left(\mathbf{u}-\mathbf{u}_{h}\right)\right\|_{L_{\infty}(D)}$, error estimator $\max _{T \in \mathcal{T}_{h}} \eta(T)$, and pollution error $\left\|\mathbf{u}-\mathbf{u}_{h}\right\|_{L_{\infty}(\Omega)}$.

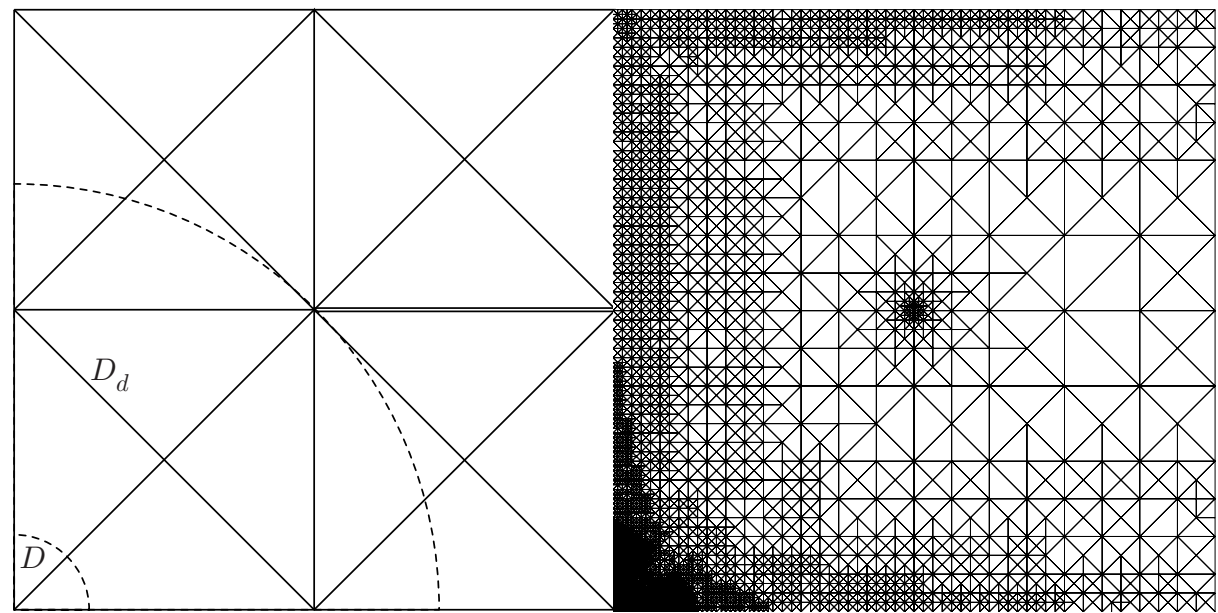

Figure 5. Initial mesh with $D=B_{1 / 4}(-1,1)$ and $D_{d}=$ $B_{\sqrt{2}}(-1,1)$ outlined (left). Computational mesh with 28974 degrees of freedom (right). 
6.3. Results. In Figure 4 we display a logarithmic error plot showing optimal-order decrease of the target error quantity $\left\|\nabla\left(\mathbf{u}-\mathbf{u}_{h}\right)\right\|_{L_{\infty}(D)}$. Note that the pollution error $\left\|\mathbf{u}-\mathbf{u}_{h}\right\|_{L_{\infty}(\Omega)}$ only decreases at the same rate as $\left\|\nabla\left(\mathbf{u}-\mathbf{u}_{h}\right)\right\|_{L_{\infty}(D)}$, which is suboptimal for the $L_{\infty}$ norm but sufficient to maintain optimality for the target error quantity. In Figure 5 we display a computational mesh having 28974 degrees of freedom. Note that the heaviest refinement occurs in the lower left hand corner in the target subdomain $D$ and also near the singularity at the origin (crack tip).

\section{ACKNOWLEDGMEnTS}

The authors would like to thank Claus-Justus Heine for providing a Stokes solver package for the ALBERTA toolbox and Jürgen Rossmann for valuable discussions concerning Lemma 10.

\section{REFERENCES}

[Ada75] R. A. Adams. Sobolev Spaces. Academic Press, New York-London, 1975. MR0450957 $(56: 9247)$

[Ama00] H. Amann. Compact embeddings of vector-valued Sobolev and Besov spaces. Glas. Mat. Ser. III, 35(55):161-177, 2000. MR1783238(2001h:46056)

[BS95] R. M. Brown and Z. Shen. Estimates for the Stokes operator in Lipschitz domains. Indiana Univ. Math. J., 44:1183-1206, 1995. MR1386766 (97c:35152)

[Dem06] A. Demlow. Localized pointwise a posteriori error estimates for gradients of piecewise linear finite element approximations to second-order quasilinear elliptic problems. SIAM J. Numer. Anal., 44:494-514, 2006. MR2218957(2007c:65105)

[Dem07] A. Demlow. Local a posteriori estimates for pointwise gradient errors in finite element methods for elliptic problems. Math. Comp., 76:19-42 (electronic), 2007. MR 2261010 (2008c:65319)

[DG10] A. Demlow and E. Georgoulis. A posteriori error estimates in the maximum norm for discontinuous Galerkin methods. Technical report, 2010.

[Fro93] S. J. Fromm. Potential space estimates for Green potentials in convex domains. Proc. Amer. Math. Soc., 119:225-233, 1993. MR1156467 (93k:35076)

[GL10] J. Guzmán and D. Leykekhman. Pointwise error estimates of finite element approximations to the Stokes problem on convex polyhedra. Technical report, 2010.

[GLRS09] J. Guzmán, D. Leykekhman, J. Rossmann, and A. H. Schatz. Hölder estimates for Green's functions on convex polyhedral domains and their applications to finite element methods. Numer. Math., 112:221-243, 2009. MR2495783(2010a:65237)

[GNS04] V. Girault, R. H. Nochetto, and L. R. Scott. Stability of the finite element Stokes projection in $W^{1, \infty}$. C. R. Math. Acad. Sci. Paris, 338:957-962, 2004. MR2066358

[GNS05] V. Girault, R. H. Nochetto, and L. R. Scott. Maximum-norm stability of the finite element Stokes projection. J. Math. Pures Appl. (9), 84:279-330, 2005. MR2121575 (2006j:76087)

[HXZL08] Y. He, J. Xu, A. Zhou, and J. Li. Local and parallel finite element algorithms for the Stokes problem. Numer. Math., 109:415-434, 2008. MR2399151 (2009j:76157)

[KMR01] V. A. Kozlov, V. G. Maz'ya, and J. Rossmann. Spectral Problems Associated with Corner Singularities of Solutions to Elliptic Equations, volume 85 of Mathematical Surveys and Monographs. American Mathematical Society, Providence, RI, 2001. MR.1788991 (2001i:35069)

[MR05] V. Maz'ya and J. Rossmann. Pointwise estimates for Green's kernel of a mixed boundary value problem to the Stokes system in a polyhedral cone. Math. Nachr., 278:17661810, 2005. MR2182091(2007b:35269)

[MR06] V. G. Maz'ya and J. Rossmann. Schauder estimates for solutions to a mixed boundary value problem for the Stokes system in polyhedral domains. Math. Methods Appl. Sci., 29:965-1017, 2006. MR2228352(2007f:35220)

[MR07] V. Maz'ya and J. Rossmann. $L_{p}$ estimates of solutions to mixed boundary value problems for the Stokes system in polyhedral domains. Math. Nachr., 280:751-793, 2007. MR2321139(2008m:35280) 
[MR10] V. G. Maz'ya and J. Rossmann. Elliptic Equations in Polyhedral Domains, volume 162 of Mathematical Surveys and Monographs. American Mathematical Society, Providence, RI, 2010. MR 2641539 (2011h:35002)

[NP94] S. A. Nazarov and B. A. Plamenevsky. Elliptic Problems in Domains with Piecewise Smooth Boundaries. Walter de Gruyter \& Co., Berlin, 1994. MR1283387 (95h:35001)

[Ros10a] J. Rossmann. Hölder estimates for Green's matrix of the Stokes system in convex polyhedra. In Around the Research of Vladimir Maz'ya II: Partial Diffential Equations, pages 315-336. Springer, 2010. MR2676181(2012a:35250)

[Ros10b] J. Rossmann. Personal communication. 2010.

[RS96] T. Runst and W. Sickel. Sobolev Spaces of Fractional Order, Nemytskij Operators, and Nonlinear Partial Differential Equations. Walter de Gruyter \& Co., Berlin, 1996. MR.1419319(98a:47071)

[SL06] E. D. Svensson and S. Larsson. Pointwise a posteriori error estimates for the Stokes equations in polyhedral domains. Preprint, 2006.

[SS05] A. Schmidt and K. G. Siebert. Design of Adaptive Finite Element Software, volume 42 of Lecture Notes in Computational Science and Engineering. Springer-Verlag, Berlin, 2005. The finite element toolbox ALBERTA, With 1 CD-ROM (Unix/Linux). MR 2127659 (2005i:65003)

[Sve06] E. D. Svensson. Computational Characterization of Mixing in Flows. PhD thesis, Chalmers University of Technology and Göteborg University, 2006. MR 2715918

[SW95] A. H. Schatz and L. B. Wahlbin. Interior maximum-norm estimates for finite element methods, Part II. Math. Comp., 64:907-928, 1995. MR 1297478 (95j:65143)

Department of Mathematics, University of Kentucky, 715 Patterson Office Tower, LeXington, KentuCKY 40506

E-mail address: alan.demlow@uky.edu

Department of Mathematical Sciences, Chalmers University of Technology and University of Gothenburg, SE-412 96 Gothenburg, Sweden

E-mail address: stig@chalmers.se 